Ann. Abeilles, I964, 7 (2), 8I-IO2.

\title{
LA PASTEURISATION DES MIELS
}

\author{
M. GONNET, P. LAVIE et J. LOUVEAUX \\ Station expérimentale d'Apicullure, \\ Centre de Recherches agronomiques du Sud-Est, Montfavet (Vaucluse) \\ Station de Recherches sur l'Abeille et les Insectes sociaux, \\ Bures-sur-Yvette (Seine-et-Oise)
}

\section{SOMMAIRE}

La pasteurisation des miels est une opération courante dans plusieurs pays et notamment au Canada et aux États-Unis. Les auteurs ont utilisé les installations de la miellerie expérimentale de l'I. N. R. A. en vue de déterminer l'intérêt de cette technique pour l'apiculture française. De nombreuses mesures et analyses ont permis d'évaluer avec précision les modifications physico-chimiques et biologiques qu'elle fait subir aux miels en fonction de leur origine et de leur composition.

\section{INTRODUCTION}

Selon les estimations les plus dignes de foi, la consommation du miel atteint en France environ 20 millions de $\mathrm{kg}$, soit, en gros, $500 \mathrm{~g}$ par personne et par an. Ce n'est donc pas un produit de première nécessité mais il est recherché pour sa supériorité sur le sucre ordinaire tant du point de vue gustatif que du point de vue diététique. Son arôme, son parfum et le fait qu'il renferme surtout des sucres simples d'assimilation facile font que, malgré son prix, un vaste public en fait une consommation régulière.

Une augmentation progressive de la consommation est souhaitée par l'ensemble des producteurs et il semble qu'elle soit assez facilement réalisable moyennant un effort publicitaire, mais on ne peut espérer la maintenir ensuite de façon durable que si, parallèlement à cet effort, on procède à une amélioration de la qualité des produits offerts au public. Or, une enquête effectuée il y a quelques années à Paris (Borneck, Louis et Louveaux, I959) a montré qu'il restait beaucoup à faire dans 
ce domaine et que beaucoup de miels commercialisés dans la capitale étaient de qualité très médiocre. C'est pourquoi, vers la même époque, nous avons entrepris d'étudier de façon systématique les méthodes permettant d'amélioter la qualité et la présentation des miels. La miellerie expérimentale que 1'I. N. R. A. installait à la Station d'Apiculture du C. R. A. du Sud-Est nous apportait les moyens matériels nécessaires à cette étude. Ce sont les résultats essentiels obtenus depuis quatre ans que nous présentons dans ce mémoire qui fait suite à celui que nous avons publié dans cette même revue (LAVIE et LoUVEAUx, I96I) pour exposer notre programme de recherches et décrire nos moyens de travail.

Pour discuter valablement de l'amélioration de la qualité des miels, il convient tout d'abord de définir les principes en fonction desquels nous entendons travailler, ce qui nous amène à préciser les critères utilisés pour juger de la qualité du miel.

Indiquons tout d'abord que nous ne prenons en considération que les facteurs de la qualité qui sont sous la dépendance du travail du producteur ou du transformateur. Ceci revient à dire que nous mettrons sur un pied d'égalité tous les miels naturels bien que, selon leur origine florale, ils présentent des caractéristiques physicochimiques et organoleptiques variables qui font qu'ils sont plus ou moins appréciés du consommateur.

On peut, à notre avis, définir comme un miel de bonne qualité un miel dont la teneur en eau n'excède pas 18,5 p. Ioo. qui ne contient pas de particules solides étrangères d'une taille supérieure à $0,2 \times 0,2 \times 0,2 \mathrm{~mm}$, dont le culot de centrifugation n'excède pas $0,35 \mathrm{ml}$ par $\mathrm{kg}$ mais qui contient cependant du pollen en quantité normale (au moins 2 ooo grains par gramme pour les miels les plus pauvres), qui ne présente aucun signe de fermentation ni aucun gôtu ou odeur parasite. I1 doit se présenter sous un aspect physique défini, soit liquide, soit finement cristallisé. Il ne doit pas présenter les signes d'un chauffage excessif (en valeur absolue et en durée) c'est-à-dire que sa teneur en hydroxyméthylfurfural (H.M.F.) doit rester inférieure à $40 \mu \mathrm{g} / \mathrm{g}$ et que l'amylase ne doit pas être détruite.

Les critères que nous venons d'énumérer nous semblent, dans l'état actuel de nos connaissances, parfaitement suffisants pour définir un produit sain, propre, stable et d'aspect agréable. Sous réserve qu'il provienne de plantes fournissant un bon nectar, il doit satisfaire les consommateurs les plus exigeants. Il peut être produit dans toute exploitation apicole travaillant avec un outillage rationnel. En effet, la teneur en eau peut être ajustée par passage des hausses en chambre chaude avant l'extraction et une propreté rigoureuse peut être obtenue par passage du miel sur des filtres très ordinaires.

Si tout apiculteur soigneux et bien outillé peut, en principe et sans difficulté technique importante préparer un miel de bonne qualité, il n'en est pas de même pour la coopérative ou pour l'industriel qui doivent travailler sur des produits de qualité variable et souvent médiocre. La préparation de gros tonnages d'un miel de qualité homogène pose des problèmes difficiles à résoudre et il est certain qu'ils ne peuvent être abordés qu'avec des méthodes appropriées. Ce sont ces méthodes, applicables essentiellement au stade du conditionnement par grosses quantités, que nous avons étudiées car ce sont elles qui, en fin de compte, conditionnent une amélioration substantielle de la qualité moyenne des miels du commerce.

Toutes ces considérations nous ont amenés à concevoir l'unité expérimentale de conditionnement du miel que nous avons installée à Montfavet comme l'outil 
répondant aux besoins de l'entreprise moyenne traitant quelques centaines de tonnes par an. L'emploi du pasteurisateur à plaques à fonctionnement continu, courant en Amérique du Nord, nous est apparu de prime abord comme la solution idéale pour l'abtention de miels répondant aux normes de qualité que nous nous sommes fixées, notamment en ce qui concerne la stabilité de l'état physique et l'absence de fermentation. L'examen des résultats obtenus jusqu'ici nous montrera si nos vues étaient justes et si l'industrie du miel peut s'orienter vers son emploi systématique sans inconvénient grave.

\section{MATÉRIEL ET MÉTHODES}

Après avoir rappelé brièvement les caractéristiques du pasteurisateur utilisé à Montfavet sans insister sur les conditions d'emploi précédemment décrites, nous indiquerons quelles sont les modifications que nous lui avons apportées depuis la rédaction de notre mémoire de 196i (LAvIE et LouVEAUX). Nous examinerons ensuite les caractéristiques des miels utilisés, puis les méthodes de prélèvement et d'analyse.

\section{A. - Matériei}

\section{1 $^{0}$ Le pasteurisateur}

Le pasteurisateur de la miellerie expérimentale de Montfavet est un appareilà plaques de marque A. P. V. utilisé ordinairement en laiterie et ne comportant aucune modification de principe. Ftant conçu comme un appareil expérimental, il comporte des dispositifs permettant de faire varier à volonté la température du circuit d'eau chaude et du circuit d'eau froide, le débit de la pompe à miel et le volume du circuit de chambrage. En agissant sur ces deux derniers facteurs, il est possible de réaliser un grand nombre de combinaisons et de faire varier très largement le débit horaire. Les contrôles de température peuvent être effectués sur tous les points importants de l'installation. La température de pasteurisation fait l'objet d'un enregistrement continu (fig. I). La pression du miel en aval de la pompe principale est constamment connue ; un pressostat limite sa valeur maximum compatible avec la résistance et l'étanchéité des canalisations, lesquelles sont en pyrex, ce qui permet de suivre sans difficulté la marche des opérations. Les prélèvements d'échantillons avant et après pasteurisation sont rendus possibles par des robinets convenablement placés.

Depuis 196I, nous avons procédé à un certain nombre d'aménagements. C'est ainsi que la température de l'eau de refroidissement est désormais réglée automatiquement par thermostat et vanne électromagnétique. On a donc supprimé la commande manuelle insuffisamment précise et susceptible de dérèglements inopinés. Le dispositif d'admission de la vapeur dans le circuit d'eau chaude a été complété par un manomètre mieux adapté et une commande directe. Un filtre à maille de $3 / 10 \mathrm{~mm}$ a été installé en amont du pasteurisateur et un manchon métallique à maille de $2 / 10 \mathrm{~mm}$ a été placé dans le chambreur ; un manchon supplémentaire élimine à la sortie de l'appareil les dernières impuretés avant la mise en pot. Énfin, un débitmètre a été installé sur la canalisation d'amenée du miel, ce qui permet de connaître par lecture directe, et non plus par le calcul, le débit de l'appareil. Il en résulte une grande simplification du réglage de la pompe d'alimentation.

Toutes ces modifications sont intervenues progressivement et ce n'est qu'en I963 que nous en avons bénéficié en totalité. Avant cette date, nous avons travaillé dans des conditions sensiblement moins favorables mais qui ne modifient en rien la valeur de l'expérimentation, sauf en ce qui concerne la filtration. Mais nous en tiendrons compte au moment de la discussion des résultats.

\section{$\left.2^{\circ}\right)$ Les miels utilisés}

L'expérimentation dont nous apportons ici les résultats porte sur i $5000 \mathrm{~kg}$ de miel environ. Aucun essai n'a porté sur moins de $500 \mathrm{~kg}$. Au total, 22 opérations de pasteurisation ont été effectuées. Le tableau I donne les principales caractéristiques de chaque lot. Signalons cependant que cinf 


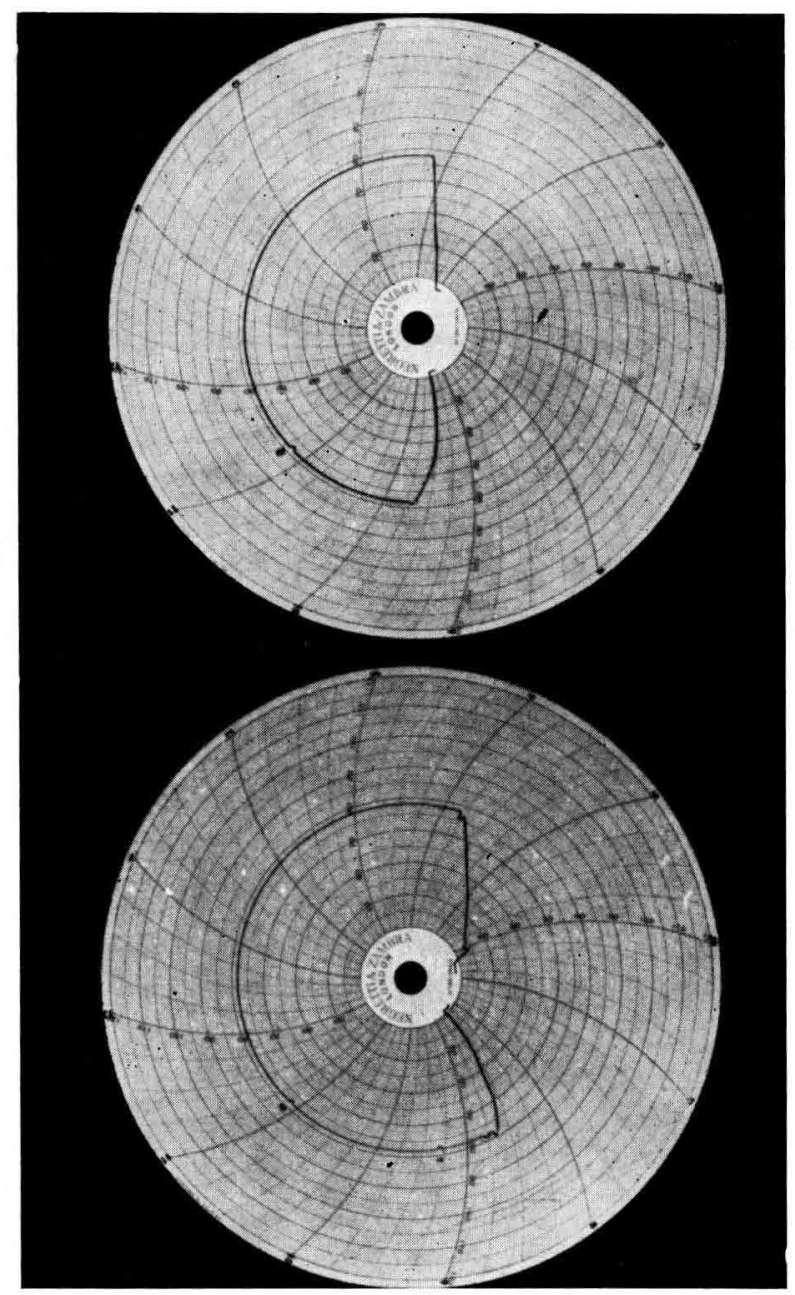

FIs. I.

Diagramme d'enregistrement de la température du miel au cours de la pasteurisation. On notera la très grande régularité du tracé.

essais de 196I ont été réalisés avec des miels qui n'ont pas été récoltés par la Station de Montfavet mais qui provenaient d'une coopérative des Landes.

Du point de vue des origines florales, on peut distinguer trois groupes :

groupe A : miel à dominance de Lavande;

groupe B : miel à dominance de Bruyère ;

groupe C : miel à dominance de Robinier faux-acacia.

\section{B. - MÉTHODES}

\section{$\mathbf{1}^{\mathrm{O}) \text { Pasteurisation }}$}

'Tous les essais ont été effectués dans les conditions normales d'utilisation industrielle de l'appareillage. On a fait varier d'un essai à l'autre la température $\left(65^{\circ}-70^{\circ}-78^{\circ}\right)$ et le temps de chambrage (2 à 15 minutes). 
TABLEAU I

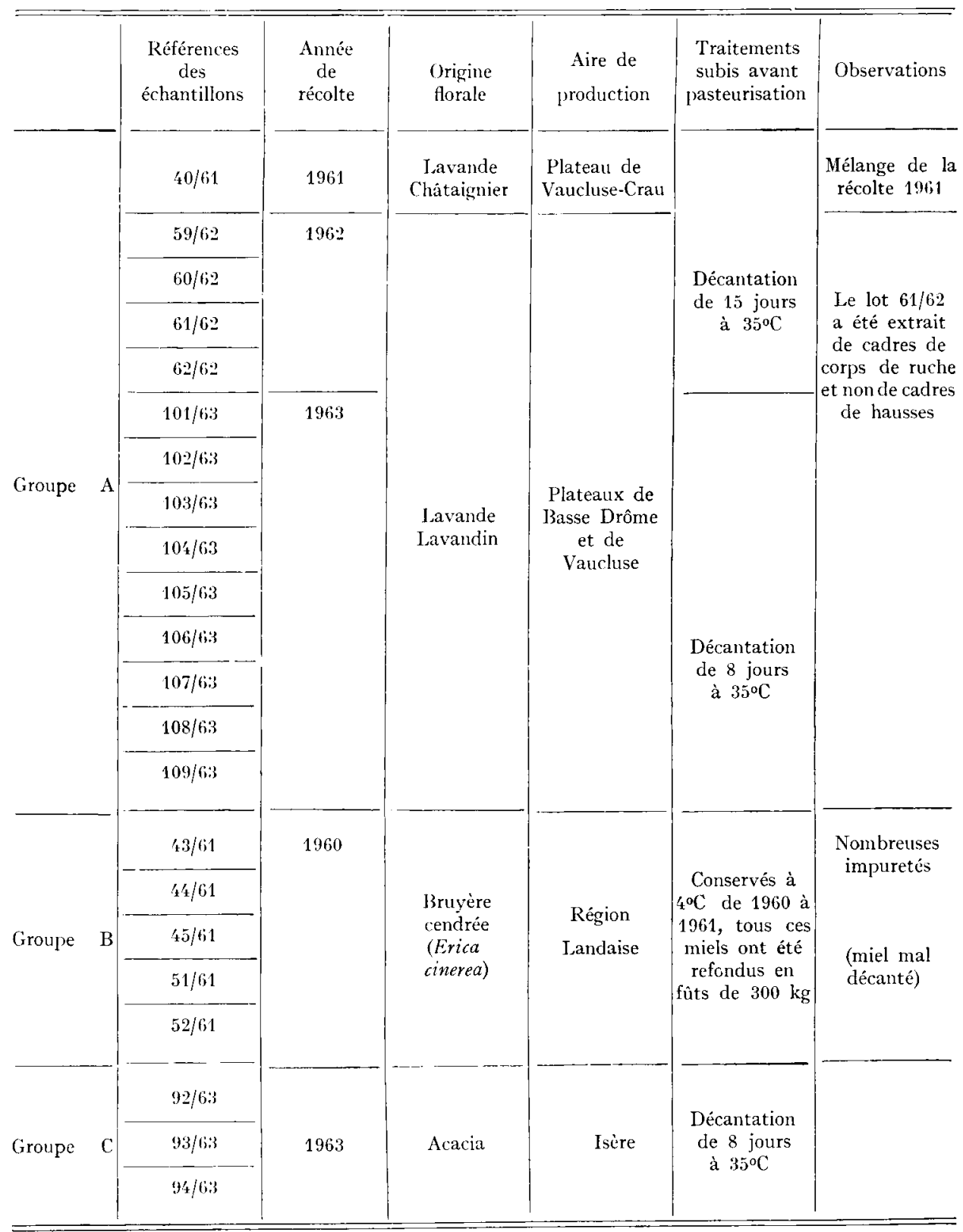




\section{$\left.2^{\circ}\right)$ Prélèvement et conservation des échantillons}

Les prélèvements sont faits avant pasteurisation au moyen d'un robinet situé à l'entrée de l'échangeur de température et après pasteurisation à l'extrémité libre du circuit. Je temps qui s'écoule entre les deux prélèvements est égal au temps de chambrage. On a ainsi la certitude que les deux échantillons sont très sensiblement comparables. On prélève 18 échantillons de $500 \mathrm{~g}$ au cours de chaque essai. Ces échantillons sont répartis comme suit :

4 échantillons pasteurisés conservés à $14^{\circ}$,

4 échantillons non pasteurisés conservés à $14^{\circ}$,

4 échantillons pasteurisés conservés à la température ordinaire du laboratoire,

4 échantillons non pasteurisés conservés à la température ordinaire du laboratoire,

I échantillon pasteurisé et

I échantillon non pasteurisé sont réservés aux contrôles immédiats. Tous les prélèvements sont faits en pots de verre soigneusement lavés, rincés à l'eau distillée et séchés à l'étuve. Les pots sont ensuite bouchés hermétiquement.

\section{$\left.3^{\circ}\right)$ Analyses et contrôles divers}

\section{a) Cristallisation.}

Pour apprécier la recristallisation, nous utilisons la méthode de White et al., (I962) à peine. modifiée, c'est-à-dire que nous nous servons de l'échelle suivante comportant des notes de o à ro:

o-- liquide limpide,

I - quelques cristaux épars,

2 - trouble léger homogène,

3 - trouble prononcé homogène,

4 - couche de cristaux de 2 à $5 \mathrm{~mm}$ d'épaisseur au fond du récipient et formation de cristaux dans la masse,

5 - couche de cristaux de 6 à 12 mm d'épaisseur au fond du récipient et formation de cristaux dans la masse,

6 - cristallisation de 25 p. Ioo de la masse du miel,

7 - cristallisation de 50 p. 100 de la masse du miel,

8 - cristallisation de 75 p. ioo de la masse du miel,

9 - cristallisation complète souple et fine,

10 - cristallisation complète dure et grossière.

\section{b) Coloration.}

Nous utilisons pour la mesure de la coloration un spectrophotomètre Jouan "Spectral-Junior " travaillant dans une bande spectrale allant de 350 à $750 \mathrm{~m} \mu$ avec une largeur de bande de $7 \mathrm{~m} \mu$. Les cuves sont des cuves parallélépipédiques de $10 \mathrm{~mm}$ d'épaisseur. Dix grammes de miel introduits dans des tubes à centrifugation sont portés à l'étuve à $70^{\circ}$ pendant 30 minutes. Ce réchauffage est indispensable pour obtenir la refonte totale des cristaux de glucose. Une centrifugation de 5 minutes à $2500 \mathrm{t} / \mathrm{mn}$ élimine toutes les bulles d'air. Le miel liquide et limpide est transvasé dans la cuve du photomètre. Ja lecture de la densité optique est faite sous 450 m $\mu$ pour les miels clairs $\left(^{1}\right)$ et sous $55^{\circ} \mathrm{m} \mu$ pour les miels foncés. Bien entendu, le même traitement s'applique à tous les échantillons même s'ils ne présentent aucune trace de cristallisation.

\section{c) Indice de réfraction.}

Les mesures sont faites au réfractomètre Abbé (marque Zeiss). La teneur en eau est obtenue en utilisant les tables de ChATAwAy (1954). Les corrections de température nécessaires sont effectuées.

d) $p H$.

La mesure du pH est faite au moyen d'un pH-mètre "Methrom " E ig6 S à électrode de verre. On utilise une solution de miel à ıo $\mathrm{p}$. Ioo dans l'eau distillée bouillie et refroidie.

(1) De o à $0,60 \mathrm{D}^{\circ}$ sous $45^{\circ} \mathrm{m} \mu$, un miel est considéré comme clair. 
e) Teneur en sucres.

Le dosage iodométrique du glucose est effectué selon la méthode de Botgaclt (I9I7). Nous travaillons sur une solution de miel à I p. Ioo et avec une prise d'essai de $10 \mathrm{ml}$. Les résultats sont exprimés en g de glucose pour ıoo grammes de miel.

On détermine le rapport glucose/eau (G/E) défini par Austin (I956) qui permet de prévoir assez bien la rapidité de recristallisation d'un miel. Les rapports inférieurs ou égaux à I, 7o correspondent à des miels à recristallisation très lente. Les rapports égaux ou supérieurs à 2 correspondent à des miels à recristallisation rapide.

La chromatographie de partage sur papier nous a permis d'effectuer un contrôle qualitatif des sucres. Nous avons utilisé la méthode de Maurizio (I959) et nous avons employé une cuve pour chromatographie descendante Shandon et du papier Wathman $n^{0} \mathrm{I}$.

f) Teneur en acides.

Nous dosons l'acidité libre, les lactones et l'acidité totale par la méthode de White et al., (1958). Les résultats sont exprimés en méq/kg de miel.

g) Hydroxyméthylfurfural (H.M.F.).

L'H.M.F. est dosé par la méthode colorimétrique qualitative et quantitative mise au point par l'un d'entre nous (GoNNET, 1963). Les résultats sont exprimés en $\mu \mathrm{g}$ par $\mathrm{g}$ de miel.

h) Invertase.

Gontarski (I957) a adapté la microméthode de dosage du glucose de Hagedorn et Jensen (1923) pour évaluer la teneur en gluco-invertase des miels. C'est cette méthode que nous avons retenue pour nos analyses. Les résultats sont exprimés en $\mathrm{mg}$ de saccharose interverti en 2 heures par la diastase contenue dans $50 \mathrm{mg}$ de miel.

i) Amylase.

La méthode que nous avons choisie est celle de Shade, Marsch et EckERT (I958). Les résultats sont exprimés en ml d'amidon (solution à $\mathrm{I}$ p. Ioo) hydrolysé en une heure par la diastase contenue dans I g de miel.

\section{j) Inhibine.}

Le dosage de l'inhibine, mis au point par Dold et Witzenhausen (I955) et repris par GonneT et LAvie (I960) a été appliqué sans modification. La souche bactérienne de référence est Bacillus subtilis CARON. Les lectures sont effectuées 24 heures après l'ensemencement et les résultats sont exprimés par une note de ò̀ 5 avec une approximation de 0,25 .

\section{k) Fermentation.}

Nous avons mis au point et utilisé pour quelques échantillons un test qui permet de reconnaître si un miel contient ou non des microorganismes vivants susceptibles de le faire fermenter. On introduit de petites quantités de miel dans des flacons stériles et on les dilue avec de l'eau stérile jusqu'à des teneurs en eau allant de 20 à $40 \mathrm{p}$. 100. Les flacons bouchés avec un tampon d'ouate stérile sont portés à l'étuve à $25^{\circ}$. On observe le développement des levures qui se traduit par un trouble de la solution et un dégagement gazeux.

\section{PRINCIPAUX RÉSULTATS}

La présentation des principaux résultats obtenus a nécessité de notre part l'établissement d'un certain nombre de tableaux qui feront l'objet d'un bref commentaire. Tous les dosages et toutes les notations ont été faits selon les principes exposés au chapitre matériel et méthodes. Il conviendra donc de se reporter à ce chapitre pour la compréhension des résultats. On consultera également le tableau I qui regroupe toutes les données relatives aux différents miels utilisés. 
I) Cristallisation (tabl. 2).

Miels $d u$ groupe $A$. - Il s'agit de miels de Lavande et Lavandin à cristallisation rapide (voir le rapport $\mathrm{G} / \mathrm{E}$ au tableau 4) ainsi qu'en témoigne le comportement des échantillons témoins. Malgré cette facilité de cristallisation, une pasteurisation à $7^{\circ}$ pendant 6 à 8 minutes est suffisante pour le maintien de l'état liquide pendant 6 mois lorsque la température de conservation est de $14^{\circ}$, et pendant

TABLEAU 2

\begin{tabular}{|c|c|c|c|c|c|c|c|c|c|c|c|c|c|c|c|}
\hline \multirow{2}{*}{ 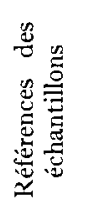 } & \multirow{2}{*}{ 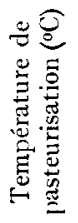 } & \multirow{2}{*}{ 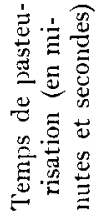 } & \multirow{2}{*}{\multicolumn{2}{|c|}{$\begin{array}{l}\text { Température de } \\
\text { conservation } \\
\text { des miels }\left({ }^{\circ}\right)\end{array}$}} & \multicolumn{11}{|c|}{ Temps de conservation } \\
\hline & & & & & 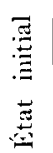 & $\stackrel{\stackrel{\infty}{9}}{\stackrel{2}{g}}$ & 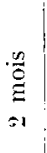 & $\begin{array}{l}\stackrel{2}{0} \\
\stackrel{\Xi}{g} \\
\therefore\end{array}$ & $\frac{\sqrt[n]{0}}{9}$ & 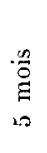 & $\begin{array}{c}\stackrel{n}{0} \\
\stackrel{2}{\Xi} \\
=\end{array}$ & 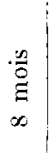 & $\begin{array}{l}\stackrel{n}{9} \\
\stackrel{g}{g}\end{array}$ & $\begin{array}{c}\frac{n}{0} \\
\stackrel{g}{g} \\
\vdots 1\end{array}$ & $\begin{array}{l}\stackrel{n}{g} \\
\stackrel{2}{g}\end{array}$ \\
\hline \multicolumn{16}{|c|}{ Groupe $A$} \\
\hline \multirow{2}{*}{$10: 3 / 63$} & \multirow{2}{*}{78} & \multirow{2}{*}{$5.2 \%$} & 1'́t & $\begin{array}{c}\text { Ténıoins } \\
\text { Pasteurisés }\end{array}$ & $\begin{array}{l}0 \\
0\end{array}$ & $\begin{array}{l}3 \\
0\end{array}$ & $\begin{array}{l}3 \\
0\end{array}$ & $\begin{array}{r}10 \\
0\end{array}$ & $\begin{array}{r}10 \\
0\end{array}$ & $\begin{array}{r}10 \\
0\end{array}$ & $\begin{array}{r}10 \\
0\end{array}$ & $\begin{array}{r}10 \\
0\end{array}$ & $\begin{array}{r}10 \\
1\end{array}$ & & \\
\hline & & & env. $: 0$ & $\begin{array}{c}\text { Témoins } \\
\text { Pasteurisés }\end{array}$ & $\begin{array}{l}0 \\
0\end{array}$ & 2 & $\begin{array}{l}3 \\
0\end{array}$ & $\begin{array}{l}3 \\
0\end{array}$ & $\begin{array}{r}10 \\
0\end{array}$ & $\begin{array}{r}10 \\
0\end{array}$ & $\begin{array}{r}10 \\
0\end{array}$ & $\begin{array}{r}10 \\
0\end{array}$ & $\begin{array}{r}10 \\
0\end{array}$ & & \\
\hline \multirow{3}{*}{$61 / 62$} & \multirow{3}{*}{78} & \multirow{3}{*}{6.28.} & 1't & $\begin{array}{c}\text { Témoins } \\
\text { Pasteurisés }\end{array}$ & $\begin{array}{l}0 \\
0\end{array}$ & $\begin{array}{l}2 \\
0\end{array}$ & $\begin{array}{l}3 \\
0\end{array}$ & $\begin{array}{l}3 \\
0\end{array}$ & $\begin{array}{l}3 \\
0\end{array}$ & $\begin{array}{l}8 \\
0\end{array}$ & $\begin{array}{l}8 \\
0\end{array}$ & $\begin{array}{l}8 \\
1\end{array}$ & $\begin{array}{l}8 \\
1\end{array}$ & $\begin{array}{l}8 \\
1\end{array}$ & $\begin{array}{l}8 \\
2\end{array}$ \\
\hline & & & חני. & Témoins & 0 & 2 & 3 & 3 & 3 & 8 & 8 & 8 & 8 & 8 & 8 \\
\hline & & & env. 20 & Pasteurisés & 0 & 0 & 0 & 0 & 0 & 0 & 0 & 0 & 0 & 1 & 1 \\
\hline \multirow{4}{*}{$61) / 62$} & \multirow{4}{*}{78} & \multirow{4}{*}{6.33} & $1_{t}^{\prime}$ & Témoins & 0 & 2 & 3 & 10) & 10 & 10 & 10 & 10 & 10 & 10 & 10 \\
\hline & & & & Pasteurisés & 0 & 0 & 0 & 0 & 0 & 0 & 0 & 1 & 1 & 1 & $\ddot{3}$ \\
\hline & & & 10 & Témoins & 0 & 2 & 3 & 3 & 10 & 10 & 10 & 10 & 10 & 10 & 10 \\
\hline & & & env. 20 & Pasteurisés & 0 & 0 & 0 & 0 & 0 & 0 & 0 & 0 & 0 & 1 & 1 \\
\hline \multirow{4}{*}{$40 / 61$} & \multirow{4}{*}{78} & \multirow{4}{*}{8.00 . } & $1 \%$ & Témoins & 0 & 3 & 9 & 9 & 9 & 9 & 9 & 9 & 9 & 9 & 9 \\
\hline & & & 1.7 & Pasteurisés & 0 & 0 & 0 & 0 & 0 & 0 & 0 & 1 & 1 & 4 & 6 \\
\hline & & & $\therefore \quad \cdot 0$ & Témoins & 0 & 3 & 9 & 9 & 9 & 9 & 9 & 9 & 9 & 9 & 9 \\
\hline & & & em. 20 & Pasteurisés & 0 & 0 & 0 & 0 & 0 & 0 & 0 & 0 & 0 & 0 & 1 \\
\hline \multirow{4}{*}{$62 / 62$} & \multirow{4}{*}{78} & \multirow{4}{*}{8.40} & 14 & Témoins & 0 & 2 & 3 & 3 & 10 & 10 & 10 & 10 & 10 & 10 & 10 \\
\hline & & & & Pasteurisés & 0 & 0 & 0 & 0 & 0 & 0 & () & 1 & 1 & 1 & 2 \\
\hline & & & & Témoins & 0 & 2 & 3 & 3 & 10 & 10 & 10 & 10 & 10 & 10 & 10 \\
\hline & & & env. 20 & Pasteurisés & 0 & 0 & 0 & 0 & 0 & 0 & 0 & 0 & 0 & 0 & 1 \\
\hline \multirow{4}{*}{$102 / 63$} & \multirow{4}{*}{78} & \multirow{4}{*}{9.48.} & 1. & Témoins & 0 & 3 & 3 & 10 & 10 & 10 & 10 & 10 & 10 & & \\
\hline & & & 14 & Pasteurisés & 0 & 0 & 0 & 0 & 0 & 0 & 1 & 2 & 3 & & \\
\hline & & & ח & Témoins & 0 & 2 & 3 & 3 & 10 & 10 & 10 & 10 & 10 & & \\
\hline & & & env. 20 & Pasteurisés & 0 & 0 & 0 & 0 & 0 & 0 & 0 & 0 & 0 & & \\
\hline
\end{tabular}


TABLEAU 2 (suite)

\begin{tabular}{|c|c|c|c|c|c|c|c|c|c|c|c|c|c|c|c|}
\hline \multirow{2}{*}{ 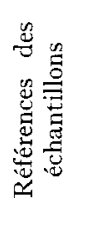 } & \multirow{2}{*}{ 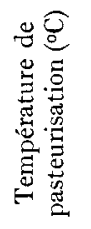 } & \multirow{2}{*}{ 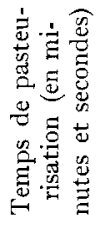 } & \multirow{2}{*}{\multicolumn{2}{|c|}{$\begin{array}{l}\text { Température de } \\
\text { conservation } \\
\text { des miels }\left({ }^{\circ} \mathrm{C}\right)\end{array}$}} & \multicolumn{11}{|c|}{ Temps de conservation } \\
\hline & & & & & 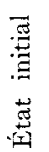 & $\begin{array}{l}\stackrel{n}{0} \\
\stackrel{0}{a} \\
-\end{array}$ & 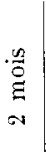 & $\stackrel{n}{\stackrel{n}{0}}$ & 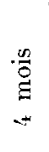 & 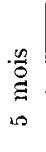 & $\begin{array}{c}0 \\
\stackrel{n}{a} \\
0\end{array}$ & $\begin{array}{l}\stackrel{n}{0} \\
\stackrel{0}{a} \\
\infty\end{array}$ & $\begin{array}{l}\cdot \frac{n \pi}{8} \\
\stackrel{g}{g} \\
\varrho\end{array}$ & 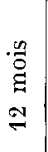 & $\begin{array}{l}\frac{.02}{3} \\
\text { g } \\
20\end{array}$ \\
\hline \multicolumn{16}{|c|}{ Groupe $A$} \\
\hline \multirow{3}{*}{$101 / 63$} & \multirow{4}{*}{78} & \multirow{4}{*}{14.10} & 14 & Témoins & 0 & 3 & 3 & 10 & 10 & 10 & 10 & 10 & 10 & & \\
\hline & & & & Pasteur & 0 & 0 & 0 & $\underline{-}$ & 0. & 0 & $\underline{0}$ & 0 & 0 & 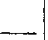 & - \\
\hline & & & env. 20 & Témoins & 0 & 2 & 3 & 3 & 10 & 10 & 10 & 10 & 10 & & \\
\hline & & & & Pasteurises & 0 & 0 & ${ }^{0}$ & 0 & - & $\stackrel{0}{-}$ & $\begin{array}{c}0 \\
\end{array}$ & $\begin{array}{c}0 \\
\end{array}$ & 0 & & - \\
\hline \multirow{4}{*}{$106 / 63$} & \multirow{4}{*}{70} & \multirow{4}{*}{5.24} & 14 & Témoins & 0 & 3 & 3 & $\begin{array}{r}10 \\
1\end{array}$ & 10 & 10 & 10 & 10 & 10 & & \\
\hline & & & & & - & - & & & - & - & - & - & - & & 一 \\
\hline & & & env, 20 & Témoins & 0 & 2 & 3 & 3 & 10 & 10 & 10 & 10 & 10 & & \\
\hline & & & & Pasteurisés & 0 & 0 & 0 & 0 & 1 & 2 & 2 & 4 & 4 & & \\
\hline \multirow{4}{*}{$105 / 63$} & \multirow{4}{*}{70} & \multirow{4}{*}{10.20} & 14 & Témoins & 0 & 3 & 3 & 10 & 10 & 10 & 10 & 10 & 10 & & \\
\hline & & & & Pasteurisés & 0 & 0 & 0 & 0 & 0 & 0 & 1 & 1 & 1 & & \\
\hline & & & & Témoins & 0 & 2 & 3 & 3 & 10 & 10 & 10 & 10 & 10 & & \\
\hline & & & env. 20 & Pasteurisés & 0 & 0 & 0 & 0 & 0 & 0 & 0 & 0 & 0 & & \\
\hline \multirow{4}{*}{$104 / 63$} & \multirow{4}{*}{70} & \multirow{4}{*}{15.18.} & & Témoins & 0 & 3 & 3 & 10 & 10 & 10 & 10 & 10 & 10 & & 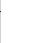 \\
\hline & & & 14 & Pasteurisés & 0 & 0 & 0 & 0 & 0 & 0 & 1 & 1 & 3 & & \\
\hline & & & & Témoins & 0 & 2 & 3 & 3 & 10 & 10 & 10 & 10 & 10 & & \\
\hline & & & env. 20 & Pasteurisés & 0 & 0 & 0 & 0 & 0 & 0 & 0 & 0 & 0 & & \\
\hline \multirow{4}{*}{$109 / 63$} & \multirow{4}{*}{65} & \multirow{4}{*}{5.23} & 14 & Témoins & 0 & 3 & 3 & 10 & 10 & 10 & 10 & 10 & 10 & & \\
\hline & & & 14 & Pasteurisés & 0 & 0 & 0 & 1 & 1 & 2 & 2 & 4 & 5 & & \\
\hline & & & ח0 זיטה & Témoins & 0 & 2 & 3 & 3 & 10 & 10 & 10 & 10 & 10 & & \\
\hline & & & env. 20 & Pasteurisés & 0 & 0 & 0 & 0 & 0 & 1 & 2 & 4 & 4 & & \\
\hline \multirow{4}{*}{$108 / 63$} & \multirow{4}{*}{65} & \multirow{4}{*}{9.33.} & $11^{2}$ & Témoins & 0 & 3 & 3 & 10 & 10 & 10 & 10 & 10 & 10 & & \\
\hline & & & Tt & Pasteurisés & 0 & 0 & 0 & 1 & 1 & 1 & 2 & 3 & 4 & & \\
\hline & & & & Témoins & 0 & 2 & 3 & 3 & 10 & 50 & 10 & $\overline{10}$ & 70 & - & $T_{1}+2$ \\
\hline & & & env. 20 & Pasteurisés & 0 & 0 & 0 & 0 & 0 & 0 & 1 & 1 & 1 & & \\
\hline & & & 14 & Témoins & 0 & 3 & 3 & 3 & 10 & 10 & 10 & 10 & 10 & 10 & 10 \\
\hline 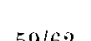 & 65 & 11 & 14 & Pasteurisés & 0 & 0 & 0 & 1 & 1 & 1 & 1 & 1 & 1 & 2 & 4 \\
\hline $50 / 02$ & (0J & $10.4 \%$ & enc 90 & Témoins & 0 & 2 & 3 & 3 & 10 & 10 & 10 & 10 & 10 & 10 & 10 \\
\hline & & & env. 20 & Pasteurisés & 0 & 0 & 0 & 1 & 1 & 1 & 1 & 1 & 1 & 1 & 2 \\
\hline & & & 14 & Témoins & 0 & 3 & 3 & 10 & 10 & 10 & 10 & 10 & 10 & & \\
\hline $107 / 63$ & 65 & 14.10 & & Pasteurisés & 0 & 0 & 0 & & 0 & 1 & 1 & 2 & 3 & & \\
\hline & & & env 20 & Témoins & 0 & 2 & 3 & 3 & 10 & 10 & 10 & 10 & 10 & & \\
\hline & & & env. 20 & Pasteurisés & 0 & 0 & 0 & 0 & 0 & 0 & 0 & 0 & 0 & & \\
\hline
\end{tabular}


TABLEAU 2 (suite)

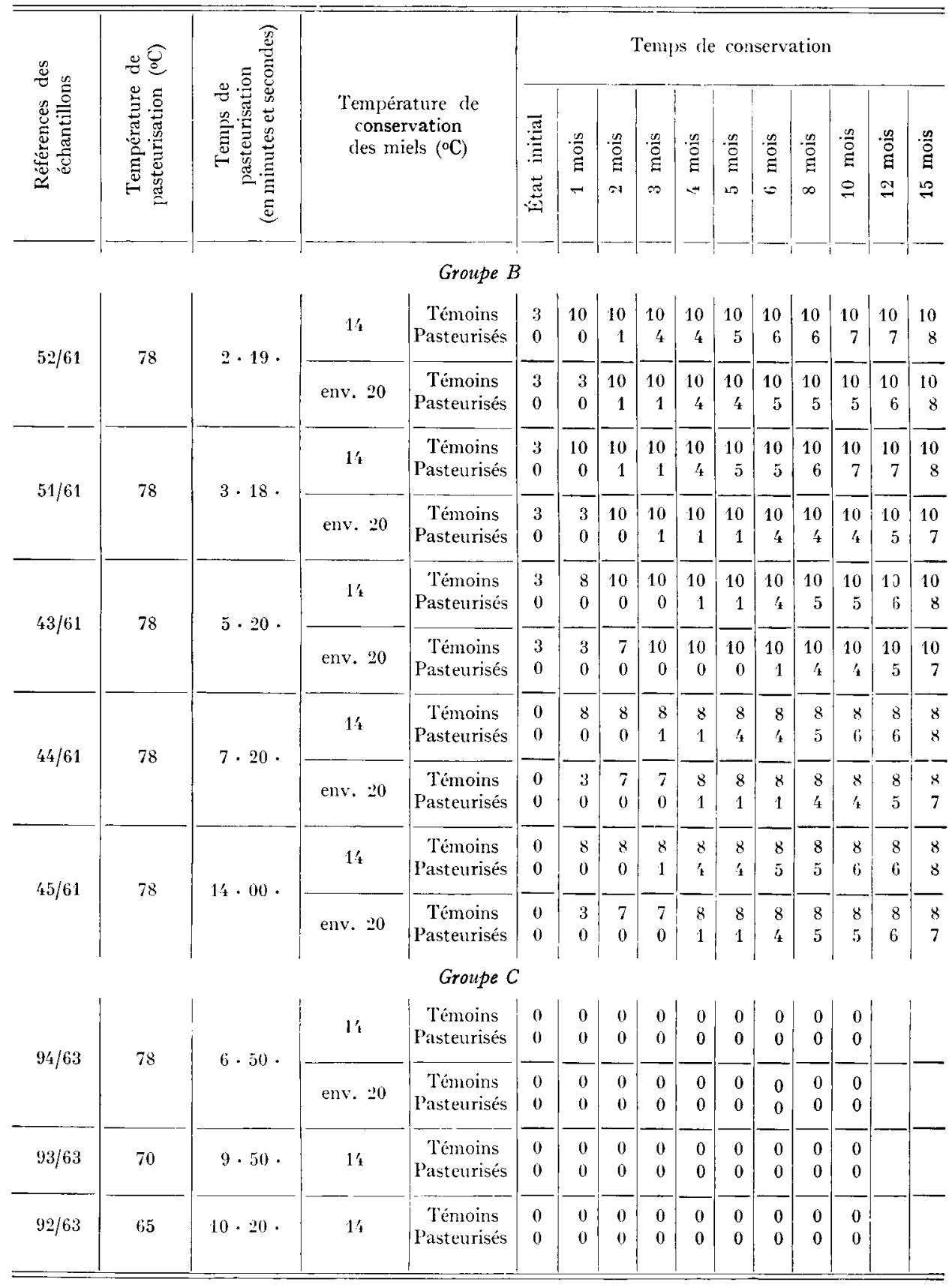


Io à I2 mois lorsqu'elle est de $20^{\circ}$ environ. Les miels ayant gardé l'état liquide pendant un an à la température de $20^{\circ}$ sont ceux qui ont été chauffés 8 minutes ou plus. Au bout de $\mathrm{I} 5$ mois de conservation, on ne note encore que quelques cristaux épars. Les essais effectués à $65^{\circ}$ montrent que cette température ne permet guère, même avec un temps de chauffage assez long, d'obtenir de bons résultats. Un début de recristallisation se manifeste généralement vers le $3^{\mathrm{e}}$ mois.

Miels du groupe B. - I s'agit de miels de Brtyère (Erica cinerea) récoltés dans les Landes et qu'il n'a pas été possible d'épurer convenablement avant la pasteurisation. Dans le cas le plus favorable, on ne dépasse pas 5 mois de conservation à l'état liquide, ce qui peut être considéré comme un échec. Cet échec est nettement imputable aux impuretés trop nombreuses qui ont pu jouer le rôle de noyaux de recristallisation.

Miels du groupe C. - Ces miels sont, par opposition à ceux du groupe A des miels à cristallisation lente. On ne pourra juger des résultats qu'après plus d'un an de conservation.

2) Coloration (tab1. 3).

On ne note aucune modification de la coloration des miels, quels qu'ils soient, sous l'influence de la pasteurisation tout au moins dans la limite de sensibilité de notre appareillage. C'est dire qu'il est impossible de noter une prise de coloration décelable à l'œil.

3) Teneur en eau (tabl. 3).

Il ressort nettement de la lecture des résultats obtenus qu'on ne peut noter aucune modification systématique de la teneur en eau sous l'influence de la pasteurisation.

4) $p H$ (tableau 3).

Le $\mathrm{pH}$ des miels n'est pas affecté par la pasteurisation. Les quelques variations observables, d'ailleurs très faibles, sont de toute évidence dues au hasard.

5) Acidité (tabl. 3).

Les quelques variations dans l'acidité totale, l'acidité libre et les lactones que l'on constate à la suite de la pasteurisation se font aussi bien dans le sens d'une augmentation que d'une diminution. Elles sont vraisemblablement en rapport avec la méthode de dosage utilisée ou avec l'hétérogénéité résiduelle des échantillons.

6) $H . M . F \cdot($ tabl. 3).

Lorsqu'il y a modification de la teneur en H.M.F., elle se fait toujours dans le sens d'une augmentation. Celle-ci atteint au maximum $5,5 \mu \mathrm{g} / \mathrm{g}$ pour les échantillons $6 \mathrm{r} / 62$ et $43 / 6 \mathrm{r}$. On peut donc la considérer comme pratiquement négligeable.

7) Sucres (tabl. 4).

Les dosages de glucose avant et après pasteurisation montrent qu'il peut y avoir une très légère modification de la teneur en glucose de certains miels. Outre que cette modification est très faible, on notera qu'il est impossible de dégager un rapport net entre l'évolution et une caractéristique donnée du miel. Par ailleurs, 
TABLEAU 3

\begin{tabular}{|c|c|c|c|c|c|c|c|c|c|c|}
\hline \multirow{2}{*}{ 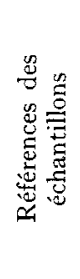 } & \multirow{2}{*}{ 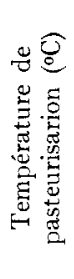 } & \multirow{2}{*}{ 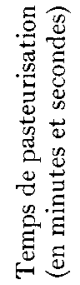 } & \multirow{2}{*}{ 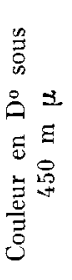 } & \multirow{2}{*}{ 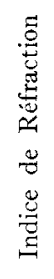 } & \multirow{2}{*}{ 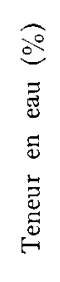 } & \multirow[b]{2}{*}{$\Xi$} & \multicolumn{3}{|c|}{ Acidités méq $/ \mathrm{kg}$} & \multirow[b]{2}{*}{ 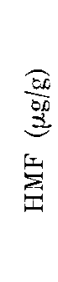 } \\
\hline & & & & & & & 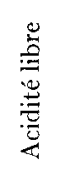 & $\begin{array}{l}\stackrel{0}{\Xi} \\
\stackrel{8}{0} \\
\stackrel{g}{=}\end{array}$ & 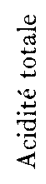 & \\
\hline
\end{tabular}

(Groupe A)

\begin{tabular}{|c|c|c|c|c|c|c|c|c|c|c|c|}
\hline $103 / 63$ & 78 & 5.02 . & $\begin{array}{l}\text { Témoins } \\
\text { Pasteurisés }\end{array}$ & $\begin{array}{l}0,36 \\
0,36\end{array}$ & $\begin{array}{l}1,4925 \\
1,4925\end{array}$ & $\begin{array}{l}17,60 \\
17,60\end{array}$ & $\begin{array}{l}3,55 \\
3,55\end{array}$ & $\begin{array}{l}26,25 \\
26\end{array}$ & $\begin{array}{l}17,50 \\
17\end{array}$ & $\begin{array}{l}43,75 \\
43\end{array}$ & $\begin{array}{l}15 \\
16\end{array}$ \\
\hline $61 / 62$ & 78 & $6 \cdot 28$. & $\begin{array}{l}\text { Témoins } \\
\text { Pasteurisés }\end{array}$ & $\begin{array}{l}0,60 \\
0,60\end{array}$ & $\begin{array}{l}1,4964 \\
1,4964\end{array}$ & $\begin{array}{l}16,05 \\
16,05\end{array}$ & $\begin{array}{l}3,90 \\
3,85\end{array}$ & $\begin{array}{l}21,75 \\
21,75\end{array}$ & $\begin{array}{l}9,75 \\
9\end{array}$ & $\begin{array}{l}31,50 \\
30,75\end{array}$ & $\begin{array}{l}27,8 \\
33\end{array}$ \\
\hline $60 / 62$ & 78 & $6 \cdot 33$. & $\begin{array}{l}\text { Témoins } \\
\text { Pasteurisés }\end{array}$ & $\begin{array}{l}0,37 \\
0,37\end{array}$ & $\begin{array}{l}1,4 \times 965 \\
1,4 \times 965\end{array}$ & $\begin{array}{l}16 \\
16\end{array}$ & $\begin{array}{l}3,70 \\
3,70\end{array}$ & $\begin{array}{l}18,75 \\
17,75\end{array}$ & $\begin{array}{l}10 \\
10,75\end{array}$ & $\begin{array}{l}28,75 \\
28,50\end{array}$ & $\begin{array}{l}20,5 \\
20,5\end{array}$ \\
\hline $40 / 61$ & 78 & 8.00 & $\begin{array}{c}\text { Témoins } \\
\text { Pasteurisés }\end{array}$ & & $\begin{array}{l}1,4957 \\
1,4+957\end{array}$ & $\begin{array}{l}16,35 \\
16,35\end{array}$ & $\begin{array}{l}4,05 \\
4,05\end{array}$ & $\begin{array}{l}30,50 \\
30,50\end{array}$ & $\begin{array}{l}12 \\
12\end{array}$ & $\begin{array}{l}42,50 \\
42,50\end{array}$ & $\begin{array}{l}14,5 \\
15,6\end{array}$ \\
\hline $62 / 62$ & 78 & $8 \cdot 40$. & $\begin{array}{l}\text { Témoins } \\
\text { Pasteurisés }\end{array}$ & $\begin{array}{l}0,46 \\
0,46\end{array}$ & $\begin{array}{l}1,4967 \\
1,4967\end{array}$ & $\begin{array}{l}15,90 \\
15,90\end{array}$ & $\begin{array}{l}3,75 \\
3,70\end{array}$ & $\begin{array}{l}19 \\
20\end{array}$ & $\begin{array}{r}10 \\
9\end{array}$ & $\begin{array}{l}29 \\
29\end{array}$ & $\begin{array}{l}19 \\
19\end{array}$ \\
\hline $102 / 63$ & 78 & $9 \cdot 48$. & $\begin{array}{l}\text { Témoins } \\
\text { Pasteurisés }\end{array}$ & $\begin{array}{l}0,36 \\
0,36\end{array}$ & $\begin{array}{l}1,4925 \\
1,4923\end{array}$ & $\begin{array}{l}17,60 \\
17,70\end{array}$ & $\begin{array}{l}3,55 \\
3,55\end{array}$ & $\begin{array}{l}26,25 \\
26,50\end{array}$ & $\begin{array}{l}17,50 \\
17,50\end{array}$ & $\begin{array}{l}43,75 \\
4^{\prime} 4\end{array}$ & $\begin{array}{l}15 \\
16\end{array}$ \\
\hline $101 / 63$ & 78 & $14 \cdot 10$ & $\begin{array}{l}\text { Témoins } \\
\text { Pasteurisés }\end{array}$ & $\begin{array}{l}0,36 \\
0,36\end{array}$ & $\begin{array}{l}1,4925 \\
1,4922\end{array}$ & $\begin{array}{l}17,60 \\
17,70\end{array}$ & $\begin{array}{l}3,55 \\
3,55\end{array}$ & $\begin{array}{l}26,25 \\
27,50\end{array}$ & $\begin{array}{l}17,50 \\
18,50\end{array}$ & $\begin{array}{l}43,75 \\
46\end{array}$ & $\begin{array}{l}15 \\
16\end{array}$ \\
\hline $106 / 63$ & 70 & $5 \cdot 24$. & $\begin{array}{l}\text { 'Témains } \\
\text { Pasteurisés }\end{array}$ & $\begin{array}{l}0,36 \\
0,36\end{array}$ & $\begin{array}{l}1,4921 \\
1,4922\end{array}$ & $\begin{array}{l}17,75 \\
17,70\end{array}$ & $\begin{array}{l}3,55 \\
3,55\end{array}$ & $\begin{array}{l}26,25 \\
27,25\end{array}$ & $\begin{array}{l}17,50 \\
17\end{array}$ & $\begin{array}{l}43,75 \\
44,25\end{array}$ & $\begin{array}{l}15 \\
15\end{array}$ \\
\hline $105 / 63$ & 70 & $10 \cdot 20$. & $\begin{array}{l}\text { Témoins } \\
\text { Pasteurisés }\end{array}$ & $\begin{array}{l}0,36 \\
0,36\end{array}$ & $\begin{array}{l}1,4921 \\
1,4922\end{array}$ & $\begin{array}{l}17,75 \\
17,70\end{array}$ & $\begin{array}{l}3,55 \\
3,55\end{array}$ & $\begin{array}{l}26,25 \\
25,50\end{array}$ & $\begin{array}{l}17,50 \\
18,50\end{array}$ & $\begin{array}{l}43,75 \\
44\end{array}$ & $\begin{array}{l}15 \\
15\end{array}$ \\
\hline $104 / 63$ & 70 & $15 \cdot 18$. & $\begin{array}{c}\text { Témoins } \\
\text { Pasteurisés }\end{array}$ & $\begin{array}{l}0,36 \\
0,36\end{array}$ & $\begin{array}{l}1,4921 \\
1,4923\end{array}$ & $\begin{array}{l}17,75 \\
17,70\end{array}$ & $\begin{array}{l}3,55 \\
3,55\end{array}$ & $\begin{array}{l}26,25 \\
26,25\end{array}$ & $\begin{array}{l}17,50 \\
17,25\end{array}$ & $\begin{array}{l}43,75 \\
43,50\end{array}$ & $\begin{array}{l}15 \\
16\end{array}$ \\
\hline $109 / 63$ & 65 & $5 \cdot 23$. & $\begin{array}{l}\text { Témoins } \\
\text { Pasteurisés }\end{array}$ & $\begin{array}{l}0,36 i \\
0,36\end{array}$ & $\begin{array}{l}1,4922 \\
1,4922\end{array}$ & $\begin{array}{l}17,70 \\
17,70\end{array}$ & $\begin{array}{l}3,55 \\
3,55\end{array}$ & $\begin{array}{l}26,25 \\
26,25\end{array}$ & $\begin{array}{l}17,50 \\
17\end{array}$ & $\begin{array}{l}43,75 \\
l_{1} 3,25\end{array}$ & $\begin{array}{l}15 \\
15\end{array}$ \\
\hline $108 / 63$ & 65 & $9 \cdot 30$. & $\begin{array}{l}\text { Témoins } \\
\text { Pasteurisés }\end{array}$ & $\begin{array}{l}0,36 \\
0,36\end{array}$ & $\begin{array}{l}1,4922 \\
1,4922\end{array}$ & $\begin{array}{l}17,70 \\
17,70\end{array}$ & $\begin{array}{l}3,55 \\
3,55\end{array}$ & $\begin{array}{l}26,25 \\
27\end{array}$ & $\begin{array}{l}17,50 \\
18\end{array}$ & $\begin{array}{l}43,75 \\
45\end{array}$ & $\begin{array}{l}15 \\
15\end{array}$ \\
\hline $59 / 62$ & 65 & 13.47 & $\begin{array}{l}\text { Témoins } \\
\text { Pasteurisés }\end{array}$ & $\begin{array}{l}0,37 \\
0,39\end{array}$ & $\begin{array}{l}1,4967 \\
1,4966\end{array}$ & $\begin{array}{l}15,90 \\
15,95\end{array}$ & $\begin{array}{l}3,65 \\
3,60\end{array}$ & $\begin{array}{l}18,50 \\
18,25\end{array}$ & $\begin{array}{l}10,50 \\
10,75\end{array}$ & $\begin{array}{l}29 \\
29\end{array}$ & $\begin{array}{l}22,7 \\
22,7\end{array}$ \\
\hline $107 / 63$ & 65 & $111 \cdot 10$. & $\begin{array}{l}\text { Témoins } \\
\text { Pasteurisés }\end{array}$ & $\begin{array}{l}0,36 \\
0,36\end{array}$ & $\begin{array}{l}1,4922 \\
1,4921\end{array}$ & $\begin{array}{l}17,70 \\
17,75\end{array}$ & $\begin{array}{l}3,55 \\
3,55\end{array}$ & $\begin{array}{l}\because 6,25 \\
\because 6,50\end{array}$ & $\begin{array}{l}17,50 \\
17,75\end{array}$ & $\begin{array}{l}43,75 \\
44,25\end{array}$ & $\begin{array}{l}15 \\
15\end{array}$ \\
\hline
\end{tabular}


TABLEAU 3 (suite)

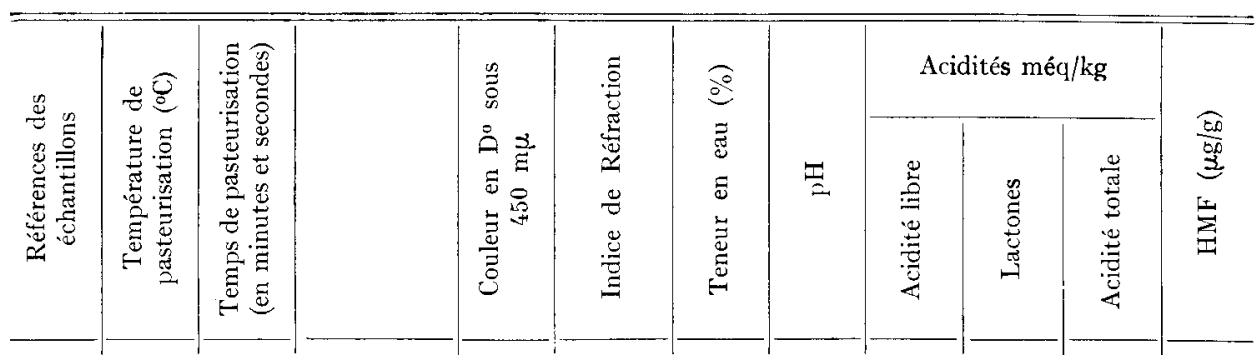

(Groupe B)

\begin{tabular}{|c|c|c|c|c|c|c|c|c|c|c|c|}
\hline $52 / 61$ & 78 & $2 \cdot 19$ & $\begin{array}{l}\text { Témoins } \\
\text { Pasteurisés }\end{array}$ & $\begin{array}{l}0, \mathbf{4} 1 \\
0,39\end{array}$ & $\begin{array}{l}1,4925 \\
1,4925\end{array}$ & $\begin{array}{l}17,60 \\
17,60\end{array}$ & $\begin{array}{l}3,90 \\
3,90\end{array}$ & $\begin{array}{l}37 \\
36,75\end{array}$ & $\begin{array}{l}25 \\
24,50\end{array}$ & $\begin{array}{l}62 \\
61,25\end{array}$ & $\begin{array}{l}22,7 \\
22,7\end{array}$ \\
\hline $51 / 61$ & 78 & $3 \cdot 18 \cdot$ & $\begin{array}{l}\text { Témoins } \\
\text { Pasteurisés }\end{array}$ & $\begin{array}{l}0,42 \\
0,40\end{array}$ & $\begin{array}{l}1,4922 \\
1,4922\end{array}$ & $\begin{array}{l}17,70 \\
17^{\circ} 70\end{array}$ & $\begin{array}{l}3,90 \\
3,90\end{array}$ & $\begin{array}{l}36,50 \\
35,75\end{array}$ & $\begin{array}{l}26 \\
26,50\end{array}$ & $\begin{array}{l}62,50 \\
62,25\end{array}$ & $\begin{array}{l}18,2 \\
20,4\end{array}$ \\
\hline $43 / 61$ & 78 & $5 \cdot 20 \cdot$ & $\begin{array}{l}\text { Témoins } \\
\text { Pasteurisés }\end{array}$ & $\begin{array}{l}0443 \\
0,42\end{array}$ & $\begin{array}{l}1,4905 \\
1,4910\end{array}$ & $\begin{array}{l}18,40 \\
18,20\end{array}$ & $\begin{array}{l}3,80 \\
3,80\end{array}$ & $\begin{array}{l}38,25 \\
38\end{array}$ & $\begin{array}{l}24,50 \\
26,50\end{array}$ & $\begin{array}{l}62,75 \\
64,50\end{array}$ & $\begin{array}{l}25 \\
30,5\end{array}$ \\
\hline $44 / 61$ & 78 & $7 \cdot 20 \cdot$ & $\begin{array}{l}\text { Témoins } \\
\text { Pasteurisés }\end{array}$ & $\begin{array}{l}0,41 \\
0,41\end{array}$ & $\begin{array}{l}1,4920 \\
1,4920\end{array}$ & $\begin{array}{l}17,80 \\
17,80\end{array}$ & $\begin{array}{l}3,90 \\
3,90\end{array}$ & $\begin{array}{l}37 \\
37,50\end{array}$ & $\begin{array}{l}25,25 \\
25,50\end{array}$ & $\begin{array}{l}62,25 \\
63\end{array}$ & $\begin{array}{l}20,5 \\
22,7\end{array}$ \\
\hline $45 / 61$ & 78 & $14 \cdot 00$ & $\begin{array}{l}\text { Témoins } \\
\text { Pasteurisés }\end{array}$ & $\begin{array}{l}0,42 \\
0,41\end{array}$ & $\begin{array}{l}1,4922 \\
1,4922\end{array}$ & $\begin{array}{l}17,70 \\
17,70\end{array}$ & $\begin{array}{l}3,90 \\
3,90\end{array}$ & $\begin{array}{l}37 \\
37,25\end{array}$ & $\begin{array}{l}25,50 \\
24,50\end{array}$ & $\begin{array}{l}62,50 \\
61,75\end{array}$ & $\begin{array}{l}25 \\
25\end{array}$ \\
\hline
\end{tabular}

(Groupe C)

\begin{tabular}{|c|c|c|c|c|c|c|c|c|c|c|c|}
\hline $94 / 63$ & $78^{\circ} \mathrm{C}$ & $6 \cdot 50$. & $\begin{array}{l}\text { Témoins } \\
\text { Pasteurisés }\end{array}$ & $\begin{array}{l}0,24 \\
0,24\end{array}$ & $\begin{array}{l}1,4941 \\
1,4941\end{array}$ & $\begin{array}{l}16,95 \\
16,95\end{array}$ & $\begin{array}{l}4,10 \\
4,10\end{array}$ & $\begin{array}{l}15,75 \\
16,25\end{array}$ & $\begin{array}{l}11 \\
11\end{array}$ & $\begin{array}{l}26,75 \\
27,25\end{array}$ & $\begin{array}{l}17 \\
19,5\end{array}$ \\
\hline $93 / 63$ & $70^{\circ} \mathrm{C}$ & $9 \cdot 50$. & $\begin{array}{l}\text { T'émoins } \\
\text { Pasteurisés }\end{array}$ & $\begin{array}{l}0,24 \\
0,24\end{array}$ & $\begin{array}{l}1,4941 \\
1,4940\end{array}$ & $\begin{array}{l}16,95 \\
17\end{array}$ & $\begin{array}{l}4,10 \\
4,10\end{array}$ & $\begin{array}{l}15,75 \\
16\end{array}$ & $\begin{array}{l}11 \\
10,50\end{array}$ & $\begin{array}{l}26,75 \\
26,50\end{array}$ & $\begin{array}{l}17 \\
19,5\end{array}$ \\
\hline $92 / 63$ & $65^{\circ} \mathrm{C}$ & $10 \cdot 20$. & $\begin{array}{l}\text { Témoins } \\
\text { Pasteurisés }\end{array}$ & $\begin{array}{l}0,24 \\
0,24\end{array}$ & $\begin{array}{l}1,49 \div 1 \\
1,4942\end{array}$ & $\begin{array}{l}16,95 \\
16,90\end{array}$ & $\begin{array}{l}4,10 \\
4,10\end{array}$ & $\begin{array}{l}15,75 \\
16\end{array}$ & $\begin{array}{l}11 \\
10,50\end{array}$ & $\begin{array}{l}26,75 \\
26,50\end{array}$ & $\begin{array}{l}17 \\
19,5\end{array}$ \\
\hline
\end{tabular}

les contrôles opérés par chromatographie sur papier n'ont pas permis de déceler de changement dans la composition en sucres des miels sous l'influence de la pasteurisation.

\section{8) Invertase (tabl. 5).}

La pasteurisation du miel provoque toujours une chute importante de l'activité de l'invertase. Cette chute est en rapport direct avec la température de pasteurisation et, d'une manière moindre, avec le temps de pasteurisation (fig. 2). On note en effet que la perte d'activité est toujours plus forte à $78^{\circ}$ qu'à $70^{\circ}$ ou $65^{\circ}$, ce qui est logique. Par contre, on constate que tous les miels ne réagissent pas de la même façon; il semble que la perte d'invertase soit d'autant plus importante que l'acidité totale est plus forte. $\mathrm{La}$ teneur en eau et le $\mathrm{pH}$ peuvent également jouer un rôle. 
TABLEAU 4

\begin{tabular}{|c|c|c|c|c|c|}
\hline $\begin{array}{l}\text { Références des } \\
\text { échantillons }\end{array}$ & $\begin{array}{l}\text { Température de } \\
\text { pasteurisation } \\
\left({ }^{\circ} \mathrm{C}\right)\end{array}$ & $\begin{array}{l}\text { Temps de pasteu- } \\
\text { risation (en mi- } \\
\text { nutes et secondes) }\end{array}$ & & $\begin{array}{l}\text { Teneur en } \\
\text { glucose }\end{array}$ & $\begin{array}{c}\text { Rapport } \\
\text { G/E }\end{array}$ \\
\hline \multicolumn{6}{|c|}{ (Groupe A) } \\
\hline $103 / 63$ & 78 & $5 \cdot 24$. & $\begin{array}{c}\text { Témoins } \\
\text { Pasteurisés }\end{array}$ & $\begin{array}{l}33,95 \\
33,30\end{array}$ & $\begin{array}{l}1,91 \\
1,89\end{array}$ \\
\hline $61 / 62$ & 78 & $6 \cdot 28$. & $\begin{array}{c}\text { Témoins } \\
\text { Pasteurisés }\end{array}$ & $\begin{array}{l}34,95 \\
34,95\end{array}$ & $\begin{array}{l}2,18 \\
2,18\end{array}$ \\
\hline $60 / 62$ & 78 & $6 \cdot 33$ & $\begin{array}{c}\text { Témoins } \\
\text { Pasteurisés }\end{array}$ & $\begin{array}{l}36,35 \\
36,35\end{array}$ & $\begin{array}{l}2,27 \\
2,27\end{array}$ \\
\hline $40 / 61$ & 78 & $8 \cdot 00 \cdot$ & $\begin{array}{c}\text { Témoins } \\
\text { Pasteurisés }\end{array}$ & & \\
\hline $62 / 62$ & 78 & $8 \cdot 40 \cdot$ & $\begin{array}{c}\text { Témoins } \\
\text { Pasteurisés }\end{array}$ & $\begin{array}{l}36,35 \\
36,35\end{array}$ & $\begin{array}{l}2,28 \\
2,98\end{array}$ \\
\hline $102 / 63$ & 78 & $9 \cdot 48$ & $\begin{array}{c}\text { Témoins } \\
\text { Pasteurisés }\end{array}$ & $\begin{array}{l}33,95 \\
33,50\end{array}$ & $\begin{array}{l}1,91 \\
1,89\end{array}$ \\
\hline $101 / 63$ & 78 & $14 \cdot 10$ & $\begin{array}{c}\text { Témoins } \\
\text { Pasteurisés }\end{array}$ & $\begin{array}{l}33,95 \\
33,30\end{array}$ & $\begin{array}{l}1,91 \\
1,88\end{array}$ \\
\hline $106 / 63$ & 70 & $5 \cdot 2{ }^{\prime}$ & $\begin{array}{c}\text { Témoins } \\
\text { Pasteurisés }\end{array}$ & $\begin{array}{l}33,95 \\
33,20\end{array}$ & $\begin{array}{l}1,91 \\
1,88\end{array}$ \\
\hline $105 / 63$ & 70 & $10 \cdot 20$ & $\begin{array}{c}\text { Témoins } \\
\text { Pasteurisés }\end{array}$ & $\begin{array}{l}33,95 \\
33,30\end{array}$ & $\begin{array}{l}1,91 \\
1,89\end{array}$ \\
\hline $104 / 63$ & 70 & $15 \cdot 18$ & $\begin{array}{c}\text { Témoins } \\
\text { Pasteurisés }\end{array}$ & $\begin{array}{l}33,95 \\
33,40\end{array}$ & $\begin{array}{l}1,91 \\
1,89\end{array}$ \\
\hline $109 / 63$ & 65 & $5 \cdot 23$ & $\begin{array}{c}\text { Témoins } \\
\text { Pasteurisés }\end{array}$ & $\begin{array}{l}33,95 \\
33,50\end{array}$ & $\begin{array}{l}1,91 \\
1,89\end{array}$ \\
\hline $108 / 63$ & 65 & $9 \cdot 33$ & $\begin{array}{c}\text { Témoins } \\
\text { Pasteurisés }\end{array}$ & $\begin{array}{l}33,95 \\
33,30\end{array}$ & $\begin{array}{l}1,91 \\
1,89\end{array}$ \\
\hline $59 / 62$ & 65 & $13 \cdot 47$. & $\begin{array}{c}\text { Témoins } \\
\text { Pasteurisés }\end{array}$ & $\begin{array}{l}35,45 \\
35,90\end{array}$ & $\begin{array}{l}2,22 \\
2,25\end{array}$ \\
\hline $107 / 63$ & 65 & $\begin{array}{l}14 \cdot 10 \cdot \\
(\text { Grou })\end{array}$ & $\begin{array}{l}\text { Témoins } \\
\text { Pasteurisés } \\
\text { C) }\end{array}$ & $\begin{array}{l}33,95 \\
33,50\end{array}$ & $\begin{array}{l}1,91 \\
1,89\end{array}$ \\
\hline $94 / 63$ & 78 & $6 \cdot 50$ & $\begin{array}{c}\text { Témoins } \\
\text { Pasteurisés }\end{array}$ & $\begin{array}{l}29,95 \\
29,80\end{array}$ & $\begin{array}{l}1,77 \\
1,76\end{array}$ \\
\hline $93 / 63$ & 70 & $9 \cdot 50$ & $\begin{array}{c}\text { Témoins } \\
\text { Pasteurisés }\end{array}$ & $\begin{array}{l}29,95 \\
29,90\end{array}$ & $\begin{array}{l}1,77 \\
1,76\end{array}$ \\
\hline $92 / 63$ & 65 & $10 \cdot 20$. & $\begin{array}{c}\text { Témoins } \\
\text { Pasteurisés }\end{array}$ & $\begin{array}{l}99,95 \\
29,60\end{array}$ & $\begin{array}{l}1,77 \\
1,75\end{array}$ \\
\hline
\end{tabular}


9) Amylase (tabl. 5).

On constate que l'amylase est beaucoup moins sensible à l'élévation de température que l'invertase. Pour atteindre une perte d'activité de 1'ordre de 50 p. roo, il faut chauffer à $7^{\circ}$. Pour un même miel, l'inactivation partielle de l'amylase est en rapport avec la température et le temps de pasteurisation (fig. 3). Pour des miels

TABLEAU 5

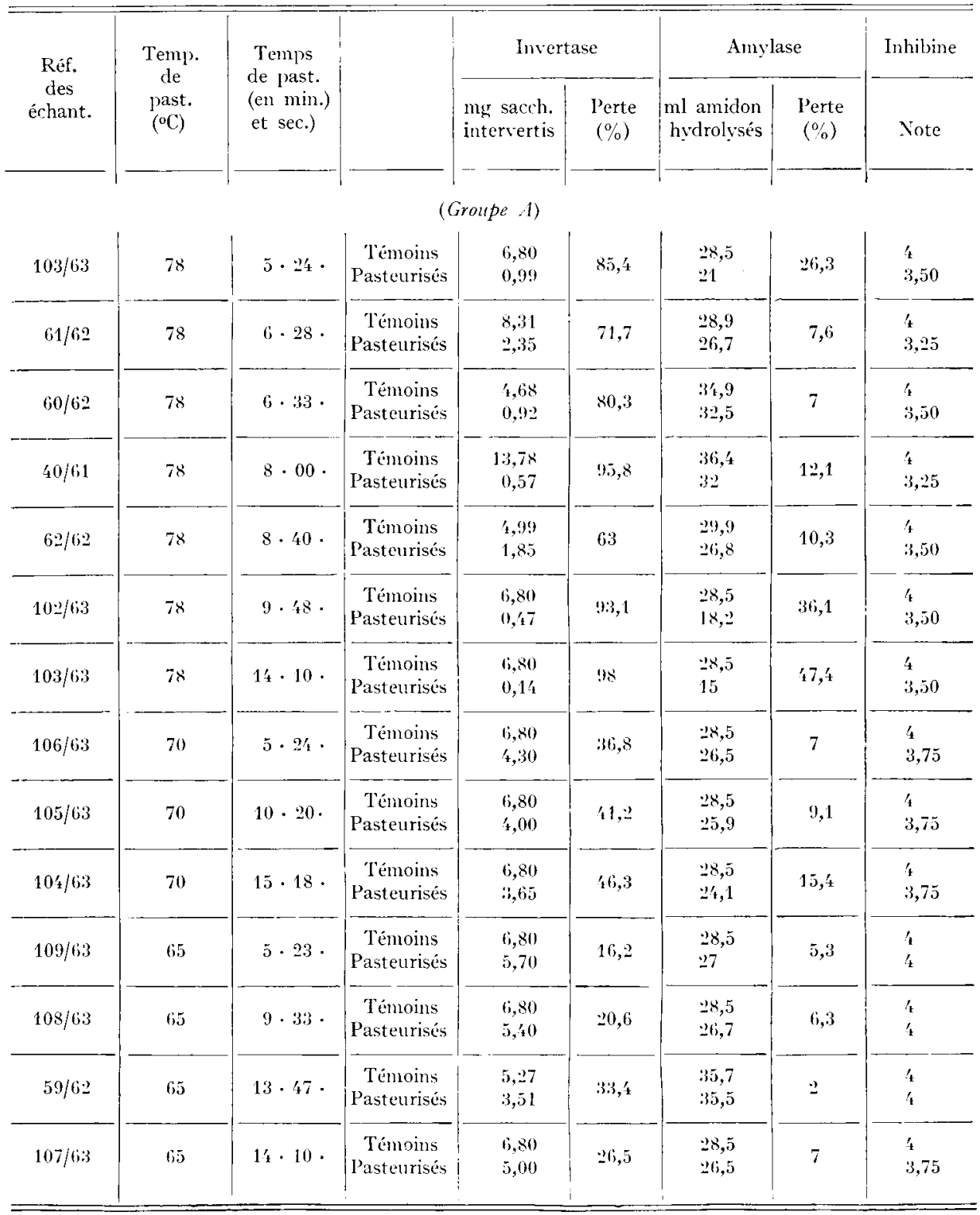


TABLEAU 5 (suite)

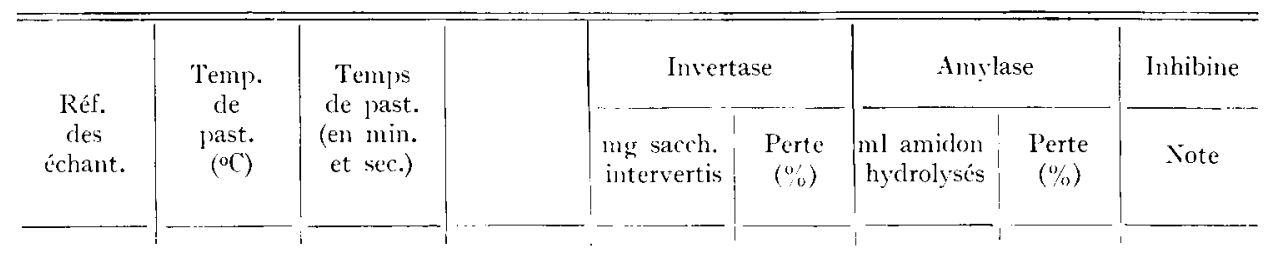

(Groupe B)

\begin{tabular}{|c|c|c|c|c|c|c|c|c|}
\hline $52 / 61$ & 78 & $-2 \cdot 19$. & $\left|\begin{array}{c}\text { T'émoins } \\
\text { Pasteurisés }\end{array}\right|$ & $\begin{array}{r}18,85 \\
2,18\end{array}$ & $8 x, 1$ & $\begin{array}{l}26,5 \\
23,7\end{array}$ & 10,5 & $\begin{array}{l}4,5 \\
4\end{array}$ \\
\hline $51 / 61$ & 78 & $3 \cdot 18$. & $\begin{array}{c}\text { T'émoins } \\
\text { P'asteurisés }\end{array}$ & $\begin{array}{r}18,28 \\
1,10\end{array}$ & 9 & $\begin{array}{l}26,1 \\
23,8\end{array}$ & 8,6 & $\begin{array}{l}4,75 \\
4\end{array}$ \\
\hline $43 / 61$ & 78 & $5 \cdot 20$. & $\begin{array}{c}\text { Témoins } \\
\text { Pasteurisés }\end{array}$ & $\begin{array}{r}1: 3,30 \\
0,95\end{array}$ & 92,8 & $\begin{array}{l}21,1 \\
20\end{array}$ & 5 & $\begin{array}{l}4,5 \\
4\end{array}$ \\
\hline $4 t / 61$ & 78 & $7 \cdot 20$. & $\begin{array}{c}\text { Témoins } \\
\text { P'asteurisés }\end{array}$ & $\begin{array}{r}19,68 \\
0,38\end{array}$ & 97 & $\begin{array}{l}21,2 \\
18,5\end{array}$ & 12,5 & $\begin{array}{l}4,75 \\
4\end{array}$ \\
\hline $45 / 61$ & 78 & $14 \cdot 00$. & $\begin{array}{c}\text { Témoins } \\
\text { Pasteurisés }\end{array}$ & $\begin{array}{r}11,73 \\
0,09\end{array}$ & 99,2 & $\begin{array}{l}21,3 \\
14\end{array}$ & 34,3 & $\begin{array}{l}4,75 \\
4\end{array}$ \\
\hline
\end{tabular}

(Groupe C)

\begin{tabular}{|c|c|c|c|c|c|c|c|c|}
\hline $9 / 163$ & 78 & $6 \cdot 50$. & $\begin{array}{c}\text { Témoins } \\
\text { Pasteurisés }\end{array}$ & $\begin{array}{l}6,00 \\
2,30\end{array}$ & $6: 3$ & $\begin{array}{l}29,6 \\
27,2\end{array}$ & 8,1 & $\begin{array}{l}\text { 't } \\
3,25\end{array}$ \\
\hline $93 / 63$ & 70 & $9 \cdot 50$. & $\begin{array}{c}\text { Témoins } \\
\text { ipasteurisés }\end{array}$ & $\begin{array}{l}6,00 \\
?, 30\end{array}$ & $2 x, 3$ & $\begin{array}{l}29,6 \\
-29\end{array}$ & 2 & $\begin{array}{l}4 \\
3,50\end{array}$ \\
\hline $92 / 63$ & 65 & $10 \cdot 20$ & $\begin{array}{c}\text { Ténoins } \\
\text { Pasteurisés }\end{array}$ & $\begin{array}{l}6,00 \\
5,05\end{array}$ & 15,1 & $\begin{array}{l}29,6 \\
29,6\end{array}$ & 0 & $\begin{array}{l}4 \\
3,50\end{array}$ \\
\hline
\end{tabular}

différents, on note une relation étroite entre la composition et le taux d'inactivation. Par exemple, l'échantillon $60 / 62$ chauffé 6 minutes $33 \mathrm{~s} \mathrm{à} 7^{\circ}$ n'a perdu que $7 \mathrm{p}$. Ioo de son amylase, perte identique à celle du miel $106 / 63$ chauffé $5 \mathrm{mn} 25 \mathrm{~s}$ à $70^{\circ}$; le miel I03/63 chauffé $5 \mathrm{mn} 25 \mathrm{~s}$ à $78^{\circ}$ a perdu par contre $26 \mathrm{p}$. Ioo de son amylase.

Il semble que les pertes les plus fortes se rencontrent chez les miels qui ont le $\mathrm{pH}$ le plus bas. Cependant, aucune relation évidente n'apparaît entre l'affaiblisse. ment du pouvoir diastasique et la teneur en eau ou l'acidité totale (tabl. 6).

Io) Inhibine (tabl. 5).

L'affaiblissement du facteur antibiotique est proportionnel à l'intensité du chautfage. La note baisse de $0,5^{\circ}$ à 0,75 point pourune pasteurisation à $78^{\circ}$, de 0,25 à 0,50 point à $70^{\circ}$ et de 0 à 0,50 point à $65^{\circ}$. Le temps de pasteurisation ne semble guère avoir d'influence sur le résultat final. Les miels dont le $\mathrm{pH}$ est le plus élevé présentent les pertes d'inhibine les plus importantes, donnée déjà acquise par GONNET et LAVIE (I960). 


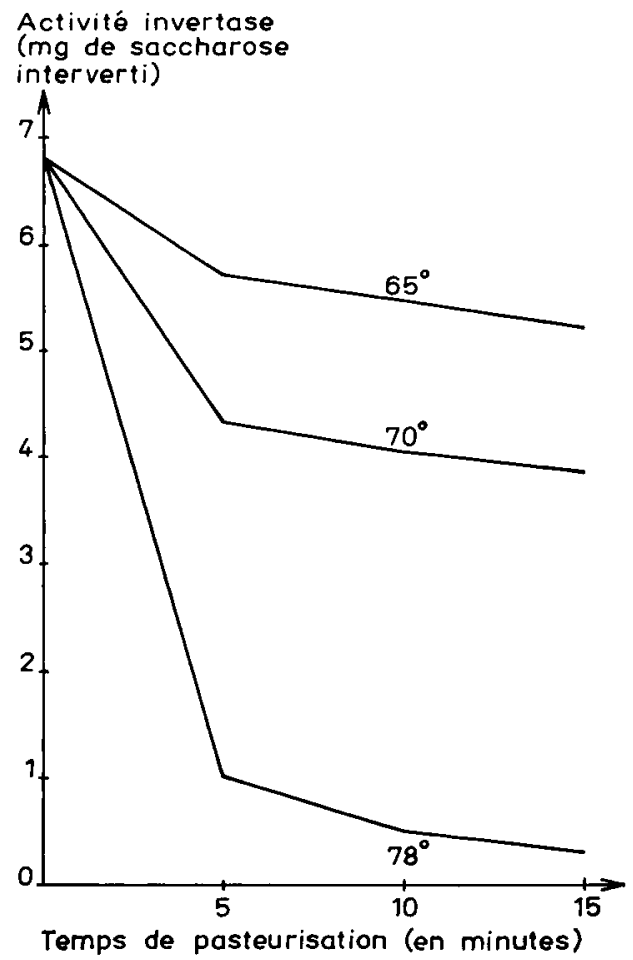

FIG. 2. - Desiruction de l'invertase par chauffage des miels de la série $\mathrm{A} / 6_{3}$.

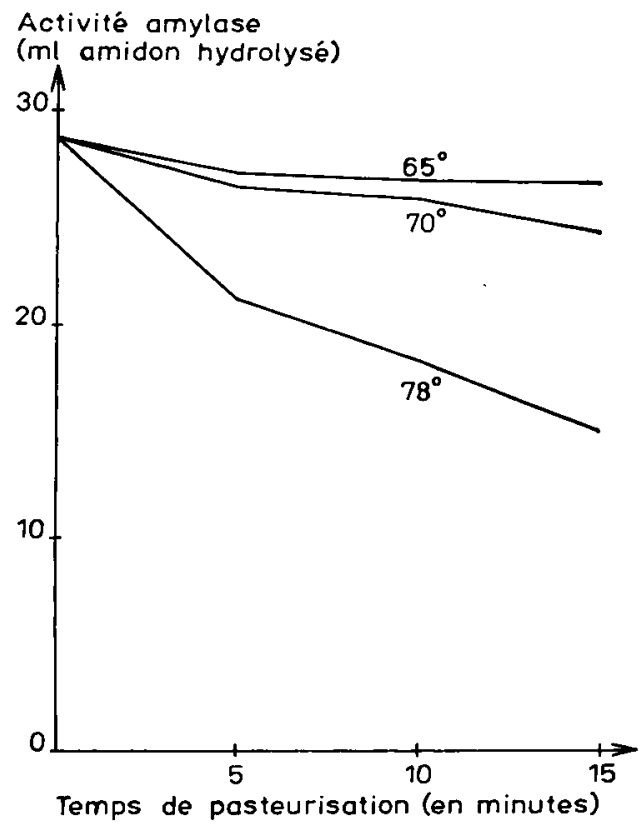

Fig. 3. - Westruction de l'amylase par chauffage des miels de la série A/63 


\section{TABLEAU 6}

\begin{tabular}{|c|c|c|c|c|c|c|c|c|}
\hline 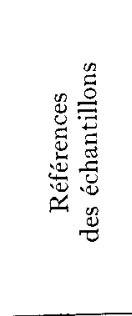 & 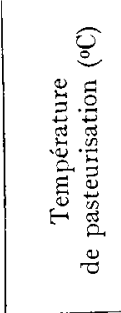 & 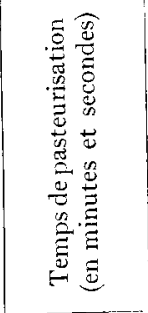 & 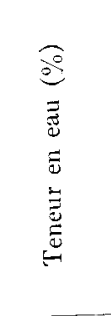 & $\equiv$ & 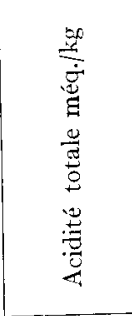 & 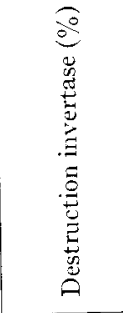 & 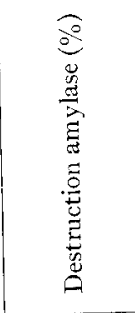 & 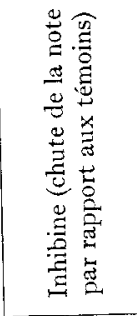 \\
\hline $52 / 61$ & 78 & $2 \cdot 19$ & 17,60 & 3,90 & 61,25 & 88,1 & $10, \tilde{5}$ & $-0,50$ \\
\hline $51 / 61$ & 78 & $3 \cdot 18$ & 17,70 & 3,90 & 62,25 & 94 & 8,6 & $-0,75$ \\
\hline $43 / 61$ & 78 & $5 \cdot 20$ & 18,20 & 3,80 & 64,50 & 92,8 & $j$ & $-0,50$ \\
\hline $103 / 63$ & 78 & $5 \cdot 2 t$ & 17,60 & 3,55 & 43 & 85,4 & 26,3 & $-0,50$ \\
\hline $61 / 62$ & 78 & $6 \cdot 28$ & 16,05 & 3,85 & 30 & 71,7 & 7,6 & $-0,75$ \\
\hline $60 / 62$ & 78 & $6 \cdot 33$ & 16 & 3,70 & 28,50 & 80,3 & 7 & $-0 ; 50$ \\
\hline $9 y / 63$ & 78 & $6 \cdot 50$ & 16,90 & 4,10 & 27,25 & 63 & 8,1 & $-0,75$ \\
\hline $4+161$ & 78 & $7 \cdot 20$ & 17,80 & 3,90 & 63 & 97 & 12,5 & $-0,75$ \\
\hline $40 / 61$ & 78 & $8 \cdot 00$ & 16,90 & 4,05 & 42,50 & 95,8 & 12,1 & $-0,75$ \\
\hline $62 / 62$ & 78 & $8 \cdot 40 \cdot$ & 15,90 & 3,75 & 29 & 63 & 10,3 & $-0,50$ \\
\hline $102 / 63$ & 78 & $9 \cdot 18$ & 17,70 & 3,55 & $4 t$ & 93,1 & 36,1 & $-0,50$ \\
\hline $45 / 61$ & 78 & $14 \cdot 00$. & 17,70 & 3,90 & 61,75 & 99,2 & 34,3 & $-0,75$ \\
\hline $101 / 63$ & 78 & $14 \cdot 10$ & 17,70 & $3,5.5$ & 16 & 98 & $4^{7}, 4$ & $-0,50$ \\
\hline $106 / 63$ & 70 & $5 \cdot 24$ & 17.70 & 3,55 & $4, \mathbf{k}, 25$ & 36,8 & 7 & $-0,25$ \\
\hline $93 / 63$ & 70 & $9 \cdot 50$ & -17 & 4,10 & 26,50 & 28,3 & 2 & $-0,50$ \\
\hline $105 / 63$ & 70 & $10 \cdot 20$ & 17,70 & 3,55 & $4^{\prime}+$ & 11,2 & 9,1 & $-0,25$ \\
\hline $104 / 63$ & 70 & $15 \cdot 18$ & 17,70 & 3,55 & 43,50 & 46,3 & 15,4 & $-0,25$ \\
\hline $109 / 63$ & 65 & $5 \cdot 23$ & 17,70 & 3,55 & 43,25 & 16,2 & 5,3 & 0 \\
\hline $108 / 63$ & 65 & $9 \cdot 33$ & 17,70 & 3,55 & 45 & 20,6 & 6,3 & 0 \\
\hline $92 / 63$ & 65 & $10 \cdot 20$ & 16,90 & 4,10 & 26,50 & 15,1 & 0 & $-0,50$ \\
\hline $59 / 62$ & 65 & $13 \cdot 47$ & 15,95 & 3,60 & 29 & 33,4 & 2 & 0 \\
\hline $107 / 63$ & 65 & $14 \cdot 10$ & 17,70 & 3,55 & 44,25 & 26,5 & 7 & $-0,25$ \\
\hline
\end{tabular}


Ir) Fermentation (tabl. 7).

Les levures du miel sont aisément détruites par chauffage. Nous n'avons pas noté de fermentation (même pour une dilution maximum à 40 p. Ioo d'eau) avec des miels pasteurisés de $2 \mathrm{mn}$ I $9 \mathrm{~s}$ à I $4 \mathrm{mn}$ Io s à $78^{\circ} \mathrm{C}$ et de $5 \mathrm{mn} 24 \mathrm{~s}$ à $\mathrm{I}_{4} \mathrm{mn}$ Io $\mathrm{s}$

TABLEAU 7

\begin{tabular}{|c|c|c|c|c|c|c|c|c|c|}
\hline \multirow{2}{*}{$\begin{array}{c}\text { Références } \\
\text { des } \\
\text { échantillons }\end{array}$} & \multirow{2}{*}{$\begin{array}{c}\text { Température } \\
\text { de } \\
\text { pasteurisation } \\
\left({ }^{\circ} \mathrm{C}\right)\end{array}$} & \multirow{2}{*}{$\begin{array}{c}\text { Temps de } \\
\text { pasteurisation } \\
\text { (en minutes } \\
\text { et secondes) }\end{array}$} & \multirow{2}{*}{$\begin{array}{l}\% \text { deau } \\
\text { dans la } \\
\text { dilution }\end{array}$} & \multicolumn{6}{|c|}{$\begin{array}{l}\text { Temps d'incubation à } 25^{\circ} \mathrm{C} \\
\text { (en jour) }\end{array}$} \\
\hline & & & & 5 & 10 & 15 & 20 & 30 & 40 \\
\hline \multicolumn{10}{|c|}{ Groupe $A$} \\
\hline $\begin{array}{c}\left({ }^{2}\right) \\
103 / 63 \\
102 / 63 \\
101 / 63\end{array}$ & 78 & $\begin{array}{r}5 \cdot 2 t \\
6 \cdot 10 . \\
14 \cdot 10 .\end{array}$ & $\begin{array}{l}20 \\
25 \\
30 \\
35 \\
40\end{array}$ & $\begin{array}{l}- \\
\cdots \\
- \\
--\end{array}$ & $\begin{array}{l}- \\
- \\
- \\
-\end{array}$ & $\begin{array}{l}- \\
- \\
- \\
-\end{array}$ & $\begin{array}{c}- \\
- \\
\cdots \\
-\end{array}$ & $\begin{array}{l}- \\
- \\
-\end{array}$ & $\begin{array}{l}- \\
- \\
-\end{array}$ \\
\hline $\begin{array}{c}\left({ }^{2}\right) \\
106 / 63 \\
105 / 63 \\
104 / 63\end{array}$ & $\{70$ & $\begin{array}{r}5 \cdot 24 . \\
10 \cdot 20 \cdot \\
15 \cdot 18 .\end{array}$ & $\begin{array}{l}20 \\
25 \\
30 \\
35 \\
40 \\
40\end{array}$ & $\begin{array}{l}- \\
- \\
-\end{array}$ & $\begin{array}{l}\overline{-} \\
- \\
- \\
-\end{array}$ & $\begin{array}{l}- \\
-- \\
- \\
-\end{array}$ & $\begin{array}{l}- \\
- \\
-\end{array}$ & $\begin{array}{l}- \\
- \\
- \\
-\end{array}$ & $\begin{array}{l}- \\
- \\
-\end{array}$ \\
\hline $109 / 63$ & 65 & $5 \cdot \geq 3$. & $\begin{array}{l}20 \\
25 \\
30 \\
35 \\
40\end{array}$ & $\begin{array}{l}- \\
- \\
-- \\
-\end{array}$ & $\begin{array}{l}- \\
- \\
-\end{array}$ & $\begin{array}{l}- \\
- \\
- \\
- \\
-\end{array}$ & $\begin{array}{l}- \\
\overline{-} \\
+\end{array}$ & $\begin{array}{l}- \\
- \\
- \\
+\end{array}$ & $\begin{array}{l}- \\
- \\
- \\
+ \\
+\end{array}$ \\
\hline $108 / 4 ; 3$ & 6.5 & $9 \cdot 333$ & $\begin{array}{l}20 \\
205 \\
35 \\
30 \\
35 \\
10\end{array}$ & $\begin{array}{l}- \\
-- \\
-- \\
- \\
-\end{array}$ & $\begin{array}{l}- \\
- \\
- \\
-\end{array}$ & $\begin{array}{l}- \\
- \\
-\end{array}$ & $\begin{array}{l}- \\
- \\
- \\
- \\
-\end{array}$ & $\begin{array}{l}- \\
- \\
- \\
+\end{array}$ & $\begin{array}{l}- \\
- \\
\overline{+} \\
+\end{array}$ \\
\hline $10-63$ & 65 & $11 \cdot 10$. & $\begin{array}{l}20 \\
20 \\
30 \\
30 \\
35 \\
40\end{array}$ & $\begin{array}{l}- \\
- \\
-\end{array}$ & $\begin{array}{l}- \\
-- \\
- \\
-\end{array}$ & $\begin{array}{l}- \\
- \\
- \\
-\end{array}$ & $\begin{array}{l}- \\
- \\
- \\
-\end{array}$ & $\begin{array}{l}- \\
- \\
- \\
+\end{array}$ & $\begin{array}{l}- \\
- \\
- \\
+\end{array}$ \\
\hline $\begin{array}{l}\text { Témoins du } \\
\text { groupe A }(\mathbf{1})\end{array}$ & & & $\begin{array}{l}20 \\
205 \\
30 \\
335 \\
40\end{array}$ & $\begin{array}{l}- \\
-- \\
- \\
-\end{array}$ & $\begin{array}{l}- \\
- \\
- \\
+\end{array}$ & $\begin{array}{l}- \\
- \\
+ \\
+ \\
+\end{array}$ & $\begin{array}{l}- \\
- \\
+ \\
+ \\
+\end{array}$ & $\begin{array}{l}- \\
+ \\
+ \\
+ \\
+\end{array}$ & $\begin{array}{l}- \\
+ \\
+ \\
+ \\
+\end{array}$ \\
\hline
\end{tabular}

(1) Tous les témoins (non pasteurisés) nous ont donné dans un mêmo groupe des résultats identiques. Chaque groupe n'occupe donc qu'une seule case.

( ${ }^{2}$ I Lorsqu'aucun développement ne s'est manifesté après 10 jours, tous les miels pasteurisés d'un même groupe ou d'une même série sont rassemblés dans une case unique.

- pas de fermentation + fermentatation 
TABLEAU 7 (suite)

\begin{tabular}{c|c}
\hline $\begin{array}{c}\text { Références } \\
\text { des } \\
\text { échantillons }\end{array}$ & $\begin{array}{c}\text { Température } \\
\text { de } \\
\text { pasteurisation } \\
\left({ }^{\circ}()\right.\end{array}$ \\
\hline
\end{tabular}

$\left({ }^{2}\right)$

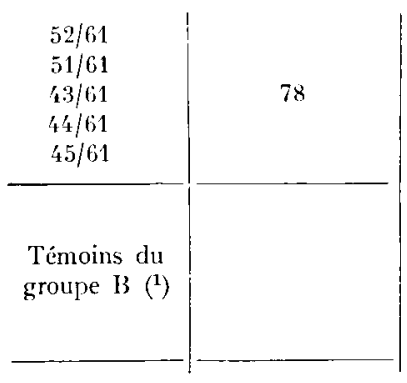

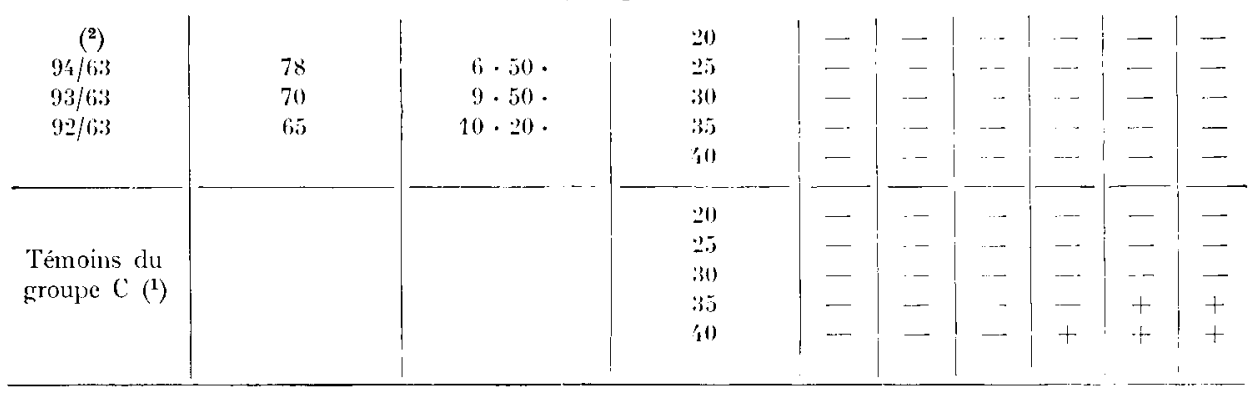

(Groupe B)

(1) Tous les témoins (non pasteurisés) nous ont donné dans un même groupe des résultats identiques. Chaque groupe n'occupe done qu'une seule case.

(2) Lorsqu'aucun développement ne s'est manifesté aprì́s 40 jours, tous les miels pasteurisés d'un môme groupe ou d'une même série sont rassemblés dans une case unique.

- pas de fermentation + fermentation

à $70^{\circ} \mathrm{C}$. Par contre, une pasteurisation à $65^{\circ} \mathrm{C}$ est insuffisante pour écarter tous risques de fermentation. Le tableau 7 donne l'ensemble des résultats obtenus.

\section{DISCUSSION}

L'ensemble des résultats obtenus jusqu'ici par la pasteurisation des miels nous fournit somme toute un tableau encourageant. Les contrôles effectués à l'heure actuelle permettent de tirer les conclusions suivantes : 
Io) Le miel pasteurisé n'est pas altéré dans l'essentiel de sa composition. Il n'y a pas formation importante d'H.M.F. comme on aurait pu le craindre. Il y a certes un affaiblissement des diastases, plus fort pour l'invertase que pour l'amylase, mais l'amylase n'est pas détruite dans des proportions telles qu'on puisse redouter une dépréciation du miel aux yeux de certains utilisateurs étrangers particulièrement pointilleux sur cette question. Le pouvoir antibactérien est à peine réduit. Sous réserve de rester dans les limites d'un temps de pasteurisation de l'ordre de 6 minutes et de ne pas dépasser $7^{\circ}$, il n'y a pas dégradation du miel. Des études sont encore nécessaires pour rechercher si une pasteurisation à moins de $7^{\circ}$ resterait suffisamment efficace. Dans l'affirmative, il serait possible de réduire encore le taux d'affaiblissement des diastases.

$2^{\circ}$ ) La pasteurisation stabilise le miel à l'état liquide pour une période de 6 à 8 mois sous réserve qu'on ne traite qu'un miel bien épuré et qu'on n'utilise que des récipients lavés. Nous insistons sur ce point car l'expérience nous a montré qu'il y a de grandes différences de stabilisation selon que l'on introduit ou non des poussières dans le miel au moment de la mise en pots.

$3^{\circ}$ ) Le miel pasteurisé est garanti contre toute fermentation ultérieure. Sa conservation peut donc se faire dans les meilleures conditions. Nous sommes donc en mesure de confirmer dans leur ensemble les résultats obtenus sur le plan technique par nos collègues nord-américains et qui ont été exposés de façon synthétique par TOWNSEND (I96I) ainsi que par MORSE (I96I). Par ailleurs, nous avons pu montrer qu'une pasteurisation conduite de façon rationnelle ne peut que valoriser les miels. Contrairement à ce qu'on pouvait craindre, elle se révèle parfaitement compatible avec les exigences de la loi française. Quelques réserves sont à faire en ce qui concerne les miels destinés à l'exportation; un affaiblissement important de l'invertase, seule conséquence notable de l'opération, risque d'être considéré actuellement dans certains pays voisins de la France comme préjudiciable à la qualité du miel. Il est d'ailleurs possible qu'à l'avenir l'attitude de ces pays se modifie sensiblement car elle repose plus sur des données subjectives que sur des faits scientifiquement établis.

L'apiculture française devrait donc pouvoir faire un usage de plus en plus important des méthodes de conditionnement du miel faisant appel à la pasteurisation, tout au moins pour le traitement de quantités importantes de produit.

$$
\text { Reçu pour publication en férier } 1964 .
$$

\author{
SUMMARY
}

PASTEURIZATION OF HONEY

The observations were conducted at Montfavet where the pasteurizer of the experimental honey processing plant has undergone certain improvements since $196 \mathrm{I}$. The series of experiments was carried out on a approximately $15,000 \mathrm{~kg}$ of honey and during its course 22 pasteurizations were effected. Analysis and inspection were carried out in accordance with the methods described. They showed that the basic composition of pasteurised honey is not altered : there is no significant H.M.F. formation, and the amylase is not sufficiently destroyed to fear degradation of the honey. Such degradation can be prevented by keeping the time of pasteurization down to about 6 minutes and the temperature below $78^{\circ} \mathrm{C}$. Pasteurization stabilizes honey in the liquid state for 6 to 8 months, provided that only suitably purified honey is treated and that the vessels are thoroughly cleaned. 


\section{RÉFÉRENCES BIBLIOGRAPHIQUES}

Austin G. H., 1956. Maltose content of canadian honeys and its probable effects on crystallisation. Tenth Intern. Congr. Entomol., 4, 1001-1006.

Borneck R., Louis J., Louveaux J., 1958. Enquête sur le marché du miel à Paris pendant l'hiver 1957-1958. Ann. Abeille, 4, 223-245.

Bougault, 1917. C.R. Acad. Sci. Fr., 164, 1008.

Chataway II. D., I954. In $A B C$ and $X Y Z$ of bee anlure, 438, Root Co, Medina, (Ohio), U. S. A.

Doly H., Witzenhausen R., 1955. Ein Verfahren zur Beurteilung der örtlichen inhibitorischen (Keimvermehrungshemmenden) Wirkung von Honigsorten verschiedener Herkunft. Z. Hyg., 141, $333-337$.

Gonnet M., 1963. L'hydroxymethylfurfural dans les miels. Mise au point d'une méthode de dosage. Ann. Abeille, 6, (1), 53-67.

Gonnet M., Lavie P., ig6o. Influence du chauffage sur le facteur antibiotique des miels. Ann. Abeille, 3, (4), 349-364.

Gontarskı H., I957. Eine Halbmikromethode zur quantitativen Bestimmung der Invertase im Bienenhonig. Z. Bienenforschung, 4, (2), 41-45.

Hagedorn et Jeasen, i923. Biochem. Z. Dtsch., 135, 46.

Lavie P., Louveaux J., i96r. La Station expérimentale d'Apiculture de l'I. N. R. A. Ann. Abeille, 4, (4), 297-360.

Maurizio A., 1959. Papierchromatographische Untersuchungen an Blutenhonigen und Nektar. Ann. Abeille, 4, 29I-34I.

Morse R. A., r96I. Honey handling in the U. S. A. P'ré-congrès scientifique. XVIII Congr. intern. Apiculture, Madrid.

Shade J., Marscir G., Eckert J., I958. Diastase activity and hydroxymethylfurfural in honey and their usefulness in detecting heat alteration. Food Research, 23, 446-463.

Townsend G. F., г96г. La préparation du miel pour la vente. Minist. agric. Ontario, 544, I-25.

White J. W., Petty J., Hager R. B., I95S. The composition of honey : II lactone content. Journ. A. O. A. C., 41, (1), 194-197.

White J. W., Riethof M. L., Subers M. H., Kushnir I., 1962. Composition of american honeys U. S. Depari. Agric. Techn. bull., 1261, I-24. 